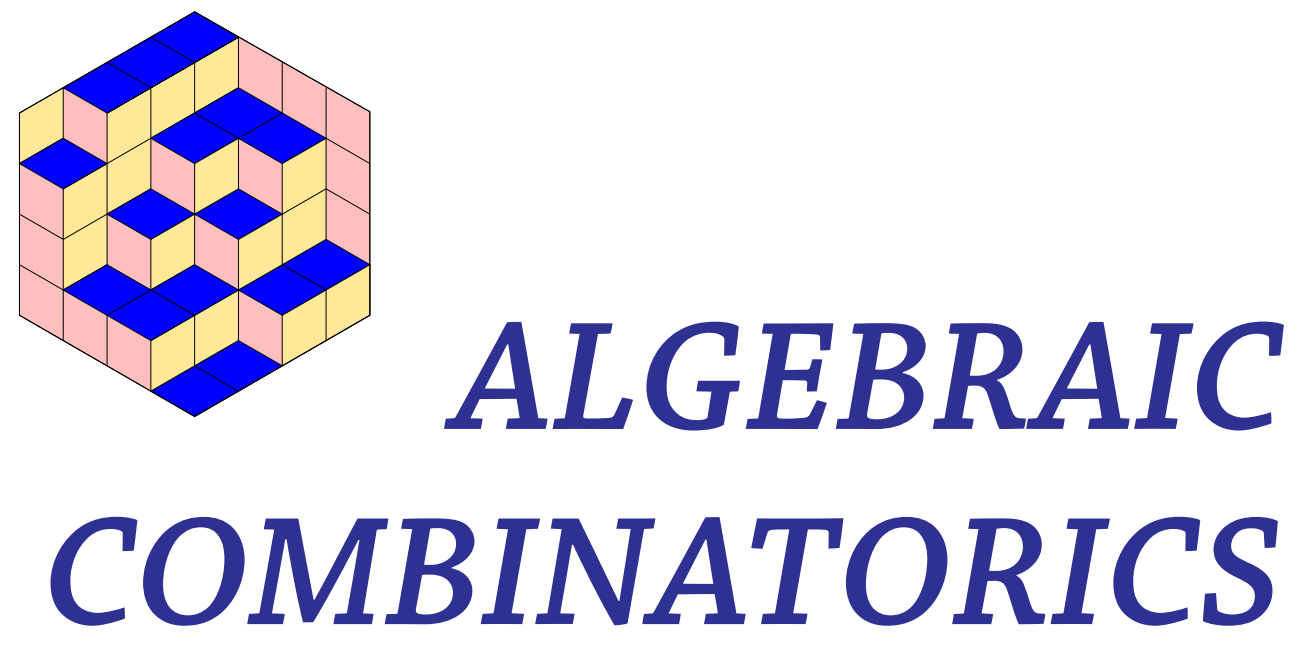

Christos A. Athanasiadis

Some applications of Rees products of posets to equivariant gamma-positivity

Volume 3, issue 1 (2020), p. 281-300.

<http://alco.centre-mersenne.org/item/ALCO_2020__3_1_281_0>

(c) The journal and the authors, 2020.

Some rights reserved.

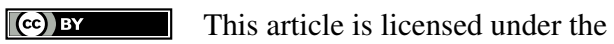

Creative Commons ATtribution 4.0 International LiCEnSE.

http://creativecommons.org/licenses/by/4.0/

Access to articles published by the journal Algebraic Combinatorics on the website http://alco.centre-mersenne.org/ implies agreement with the Terms of Use (http://alco.centre-mersenne.org/legal/).

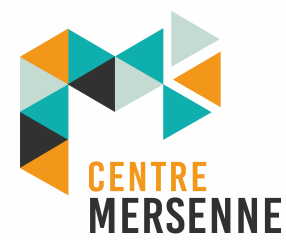

Algebraic Combinatorics is member of the Centre Mersenne for Open Scientific Publishing www.centre-mersenne.org 


\title{
Some applications of Rees products of posets to equivariant gamma-positivity
}

\author{
Christos A. Athanasiadis
}

\begin{abstract}
The Rees product of partially ordered sets was introduced by Björner and Welker. Using the theory of lexicographic shellability, Linusson, Shareshian and Wachs proved formulas, of significance in the theory of gamma-positivity, for the dimension of the homology of the Rees product of a graded poset $P$ with a certain $t$-analogue of the chain of the same length as $P$. Equivariant generalizations of these formulas are proven in this paper, when a group of automorphisms acts on $P$, and are applied to establish the Schur gamma-positivity of certain symmetric functions arising in algebraic and geometric combinatorics.
\end{abstract}

\section{INTRODUCTION}

The Rees product $P * Q$ of two partially ordered sets (posets, for short) was introduced and studied by Björner and Welker [8] as a combinatorial analogue of the Rees construction in commutative algebra (a precise definition of $P * Q$ can be found in Section 2). The connection of the Rees product of posets to enumerative combinatorics was hinted in $[8$, Section 5], where it was conjectured that the dimension of the homology of the Rees product of the truncated Boolean algebra $B_{n} \backslash\{\varnothing\}$ of rank $n-1$ with an $n$-element chain equals the number of permutations of $[n]:=\{1,2, \ldots, n\}$ without fixed points. This statement was generalized in several ways in [17], using enumerative and representation theoretic methods, and in [12], using the theory of lexicographic shellability.

One of the results of [12] proves formulas [12, Corollary 3.8] for the dimension of the homology of the Rees product of an EL-shellable poset $P$ with a contractible poset which generalizes the chain of the same rank as $P$. This paper provides an equivariant analogue of this result which seems to have enough applications on its own to be of independent interest. To state it, let $P$ be a finite bounded poset, with minimum element $\hat{0}$ and maximum element $\hat{1}$, which is graded of rank $n+1$, with rank function $\rho: P \rightarrow\{0,1, \ldots, n+1\}$ (for basic terminology on posets, see [26, Chapter 3]). Fix a field $\mathbf{k}$ and let $G$ be a finite group which acts on $P$ by order preserving bijections. Then, $G$ defines a permutation representation $\alpha_{P}(S)$ over $\mathbf{k}$ for every $S \subseteq[n]$, induced by the action of $G$ on the set of maximal chains of the rank-selected subposet

$$
P_{S}=\{x \in P: \rho(x) \in S\} \cup\{\hat{0}, \hat{1}\}
$$

Manuscript received 19th July 2018, revised 30th April 2019, accepted 6th May 2019.

KEYWORDS. Rees product, poset homology, group action, Schur gamma-positivity, local face module. 
of $P$. Following Stanley's seminal work [21], we may consider the virtual $G$ representation

$$
\beta_{P}(S)=\sum_{T \subseteq S}(-1)^{|S-T|} \alpha_{P}(T),
$$

defined equivalently by the equations

$$
\alpha_{P}(T)=\sum_{S \subseteq T} \beta_{P}(S)
$$

for $T \subseteq[n]$. The dimensions of $\alpha_{P}(S)$ and $\beta_{P}(S)$ are important enumerative invariants of $P$, known as the entries of its flag $f$-vector and flag $h$-vector, respectively. When $P$ is Cohen-Macaulay over $\mathbf{k}, \beta_{P}(S)$ is isomorphic to the non-virtual $G$-representation $\widetilde{H}_{|S|-1}\left(\bar{P}_{S} ; \mathbf{k}\right)$ induced on the top homology group of $\bar{P}_{S}:=P_{S} \backslash\{\hat{0}, \hat{1}\}[21$, Theorem 1.2]. As discussed and illustrated in various situations in [21], the decomposition of $\beta_{P}(S)$ as a direct sum of irreducible $G$-representations often leads to very interesting refinements of the flag $h$-vector of $P$.

As in references $[12,17]$, we write $\beta(\bar{P})$ in place of $\beta_{P}([n])$ and note, as just mentioned, that this $G$-representation is isomorphic to $\widetilde{H}_{n-1}(\bar{P} ; \mathbf{k})$ if $P$ is CohenMacaulay over $\mathbf{k}$. We denote by $T_{t, n}$ the poset whose Hasse diagram is a complete $t$-ary tree of height $n$, rooted at the minimum element. We denote by $P^{-}, P_{-}$and $\bar{P}$ the poset obtained from $P$ by removing its maximum element, or minimum element, or both, respectively, and recall from [17] (see also Section 2) that the action of $G$ on $P$ induces actions on $P^{-} * T_{t, n}$ and $\bar{P} * T_{t, n-1}$ as well. We also write $[a, b]:=\{a, a+1, \ldots, b\}$ for integers $a \leqslant b$ and denote by $\operatorname{Stab}(\Theta)$ the set of all subsets, called stable, of $\Theta \subseteq \mathbb{Z}$ which do not contain two consecutive integers. The following result reduces to $[12$, Corollary 3.8], proven in [12] under additional shellability assumptions on $P$, in the special case of a trivial action.

THEOREM 1.1. Let $G$ be a finite group acting on a finite bounded graded poset $P$ of rank $n+1$ by order preserving bijections. Then,

$$
\begin{aligned}
& \beta\left(\left(P^{-} * T_{t, n}\right)_{-}\right) \cong{ }_{G \in \operatorname{Stab}([n-1])} \beta_{P}([n] \backslash S) t^{|S|}(1+t)^{n-2|S|} \\
& \quad+\sum_{S \in \operatorname{Stab}([n-2])} \beta_{P}([n-1] \backslash S) t^{|S|+1}(1+t)^{n-1-2|S|}
\end{aligned}
$$

and

$$
\text { (5) } \begin{aligned}
\beta\left(\bar{P} * T_{t, n-1}\right) \cong{ }_{G} \sum_{S \in \operatorname{Stab}([2, n-2])} & \beta_{P}([n-1] \backslash S) t^{|S|+1}(1+t)^{n-2-2|S|} \\
& +\sum_{S \in \operatorname{Stab}([2, n-1])} \beta_{P}([n] \backslash S) t^{|S|}(1+t)^{n-1-2|S|}
\end{aligned}
$$

for every positive integer $t$, where $\cong_{G}$ stands for isomorphism of $G$-representations. If $P$ is Cohen-Macaulay over $\mathbf{k}$, then the left-hand sides of (4) and (5) may be replaced by the G-representations $\widetilde{H}_{n-1}\left(\left(P^{-} * T_{t, n}\right)_{-} ; \mathbf{k}\right)$ and $\widetilde{H}_{n-1}\left(\bar{P} * T_{t, n-1} ; \mathbf{k}\right)$, respectively, and all representations which appear in these formulas are non-virtual.

Several applications of [12, Corollary 3.8] to $\gamma$-positivity appear in [3, Section 3] [12] and are summarized in [5, Section 2.4]. Theorem 1.1 has non-trivial applications to Schur $\gamma$-positivity, which we now briefly discuss. A polynomial in $t$ with real coefficients is said to be $\gamma$-positive if for some $m \in \mathbb{N}$, it can be written as a nonnegative linear combination of the binomials $t^{i}(1+t)^{m-2 i}$ for $0 \leqslant i \leqslant m / 2$. Clearly, all such polynomials have symmetric and unimodal coefficients. Two symmetric function 
identities due to Gessel (unpublished), stated without proof in [12, Section 4] [18, Theorem 7.3], can be written in the form

$$
\frac{1-t}{E(\mathbf{x} ; t z)-t E(\mathbf{x} ; z)}=1+\sum_{n \geqslant 2} z^{n} \sum_{k=0}^{\lfloor(n-2) / 2\rfloor} \xi_{n, k}(\mathbf{x}) t^{k+1}(1+t)^{n-2 k-2}
$$

and

$$
\frac{(1-t) E(\mathbf{x} ; t z)}{E(\mathbf{x} ; t z)-t E(\mathbf{x} ; z)}=1+\sum_{n \geqslant 1} z^{n} \sum_{k=0}^{\lfloor(n-1) / 2\rfloor} \gamma_{n, k}(\mathbf{x}) t^{k+1}(1+t)^{n-1-2 k},
$$

where $E(\mathbf{x} ; z)=\sum_{n \geqslant 0} e_{n}(\mathbf{x}) z^{n}$ is the generating function for the elementary symmetric functions in $\mathbf{x}=\left(x_{1}, x_{2}, \ldots\right)$ and the $\xi_{n, k}(\mathbf{x})$ and $\gamma_{n, k}(\mathbf{x})$ are Schur-positive symmetric functions, whose coefficients in the Schur basis can be explicitly described (see Corollary 4.1). The coefficients of $z^{n}$ in the right-hand sides of Equations (6) and (7) are Schur $\gamma$-positive symmetric functions, in the sense that their coefficients in the Schur basis are $\gamma$-positive polynomials in $t$ with the same center of symmetry. Their Schur $\gamma$-positivity refines the $\gamma$-positivity of derangement and Eulerian polynomials, respectively; see [5, Section 2.5] and Corollary 4.1 for more information.

We will show (see Section 4) that Gessel's identities can in fact be derived from the special case of Theorem 1.1 in which $P^{-}$is the Boolean algebra $B_{n}$, endowed with the natural symmetric group action. Moreover, applying the theorem when $P^{-}$ is a natural signed analogue of $B_{n}$, endowed with a hyperoctahedral group action, we obtain new identities of the form

$$
\frac{E(\mathbf{x} ; t z)-t E(\mathbf{x} ; z)}{E(\mathbf{x} ; t z) E(\mathbf{y} ; t z)-t E(\mathbf{x} ; z) E(\mathbf{y} ; z)}=\sum_{n \geqslant 0} z^{n} \sum_{k=0}^{\lfloor n / 2\rfloor} \xi_{n, k}^{+}(\mathbf{x}, \mathbf{y}) t^{k}(1+t)^{n-2 k}
$$

$$
\frac{E(\mathbf{x} ; z)-E(\mathbf{x} ; t z)}{E(\mathbf{x} ; t z) E(\mathbf{y} ; t z)-t E(\mathbf{x} ; z) E(\mathbf{y} ; z)}=\sum_{n \geqslant 1} z^{n} \sum_{k=0}^{\lfloor(n-1) / 2\rfloor} \xi_{n, k}^{-}(\mathbf{x}, \mathbf{y}) t^{k}(1+t)^{n-1-2 k}
$$

$$
\frac{E(\mathbf{x} ; z) E(\mathbf{x} ; t z)(E(\mathbf{y} ; t z)-t E(\mathbf{y} ; z))}{E(\mathbf{x} ; t z) E(\mathbf{y} ; t z)-t E(\mathbf{x} ; z) E(\mathbf{y} ; z)}=\sum_{n \geqslant 0} z^{n} \sum_{k=0}^{\lfloor n / 2\rfloor} \gamma_{n, k}^{+}(\mathbf{x}, \mathbf{y}) t^{k}(1+t)^{n-2 k}
$$

and

$$
\frac{t E(\mathbf{x} ; z) E(\mathbf{x} ; t z)(E(\mathbf{y} ; z)-E(\mathbf{y} ; t z))}{E(\mathbf{x} ; t z) E(\mathbf{y} ; t z)-t E(\mathbf{x} ; z) E(\mathbf{y} ; z)}=\sum_{n \geqslant 1} z^{n} \sum_{k=1}^{\lfloor(n+1) / 2\rfloor} \gamma_{n, k}^{-}(\mathbf{x}, \mathbf{y}) t^{k}(1+t)^{n+1-2 k}
$$

where the $\xi_{n, k}^{ \pm}(\mathbf{x}, \mathbf{y})$ and $\gamma_{n, k}^{ \pm}(\mathbf{x}, \mathbf{y})$ are Schur-positive symmetric functions in the sets of variables $\mathbf{x}=\left(x_{1}, x_{2}, \ldots\right)$ and $\mathbf{y}=\left(y_{1}, y_{2}, \ldots\right)$ separately. Their Schur positivity refines the $\gamma$-positivity of type $B$ analogues or variants of derangement and Eulerian polynomials; this is explained and generalized in the sequel [6] to this paper. Note that for $\mathbf{x}=0$, the left-hand side of Equation (8) specializes to that of (6) (with $\mathbf{x}$ replaced by $\mathbf{y})$ and the sum of the left-hand sides of Equations (10) and (11) specializes to that of (7) (again with $\mathbf{x}$ replaced by $\mathbf{y}$ ).

Various combinatorial and algebraic-geometric interpretations of the left-hand sides of Equations (6) and (7) are discussed in [12, Section 4] [18, Section 7] [19]. For instance, by [23, Proposition 4.20], the coefficient of $z^{n}$ in the left-hand side of (6) can be interpreted as the Frobenius characteristic of the symmetric group representation 
on the local face module of the barycentric subdivision of the $(n-1)$-dimensional simplex, twisted by the sign representation. Thus, the Schur $\gamma$-positivity of this coefficient, manifested by Equation (6), is an instance of the local equivariant Gal phenomenon, as discussed in [5, Section 5.2]. Section 5 shows that another instance of this phenomenon follows from the specialization $\mathbf{x}=\mathbf{y}$ of Equation (8). Similarly, setting $\mathbf{y}=0$ to (10) yields another identity, recently proven by Shareshian and Wachs (see Proposition 3.3 and Theorem 3.4 in [20]) in order to establish the equivariant Gal phenomenon for the symmetric group action on the $n$-dimensional stellohedron and Section 6 combines Equation (6) with (10) to establish the same phenomenon for the hyperoctahedral group action on its associated Coxeter complex. Further applications of Theorem 1.1 are given in [6]. It would be interesting to find direct combinatorial proofs of Equations (8)-(11) and to generalize other known interpretations of the left-hand sides of Equations (6) and (7) to those of (8)-(11).

OutLine. The proof of Theorem 1.1 is given in Section 3, after the relevant background and definitions are explained in Section 2. This proof is fairly elementary and different from that of [12, Corollary 3.8]. Section 4 derives Equations (6)-(11) from Theorem 1.1 and provides explicit combinatorial interpretations, in terms of standard Young (bi)tableaux and their descents, for the Schur-positive symmetric functions which appear there. Sections 5 and 6 provide the promised applications of Equations (8) and (10) to the equivariant $\gamma$-positivity of the symmetric group representation on the local face module of a certain triangulation of the simplex and the hyperoctahedral group representation on the cohomology of the projective toric variety associated to the Coxeter complex of type $B$.

\section{Preliminaries}

This section briefly records definitions and background on posets, group representations and (quasi)symmetric functions. For basic notions and more information on these topics, the reader is referred to the sources [15, 21] [25, Chapter 7] [26, Chapter 3] [30]. The symmetric group of permutations of the set $[n]$ is denoted by $\mathfrak{S}_{n}$ and the cardinality of a finite set $S$ by $|S|$.

2.1. Group actions on posets and Rees products. All groups and posets considered here are assumed to be finite. Homological notions for posets always refer to those of their order complex; see [30, Lecture 1]. A poset $P$ has the structure of a $G$-poset if the group $G$ acts on $P$ by order preserving bijections. Then, $G$ induces a representation on every reduced homology group $\widetilde{H}_{i}(P ; \mathbf{k})$, for every field $\mathbf{k}$.

Suppose that $P$ is a $G$-poset with minimum element $\hat{0}$ and maximum element 1. Sundaram [29] (see also [30, Theorem 4.4.1]) established the isomorphism of $G$ representations

$$
\bigoplus_{k \geqslant 0}(-1)^{k} \bigoplus_{x \in P / G} \widetilde{H}_{k-2}((\hat{0}, x) ; \mathbf{k}) \uparrow_{G_{x}}^{G} \cong_{G} 0 .
$$

Here $P / G$ stands for a complete set of $G$-orbit representatives, $(\hat{0}, x)$ denotes the open interval of elements of $P$ lying strictly between $\hat{0}$ and $x, G_{x}$ is the stabilizer of $x$ and $\uparrow$ denotes induction. Moreover, $\widetilde{H}_{k-2}((\hat{0}, x) ; \mathbf{k})$ is understood to be the trivial representation $1_{G_{x}}$ if $x=\hat{0}$ and $k=0$, or $x$ covers $\hat{0}$ and $k=1$.

The Lefschetz character of a finite $G$-poset $P$ (over the field $\mathbf{k}$ ) is defined as the virtual $G$-representation

$$
L(P ; G):=\bigoplus_{k \geqslant 0}(-1)^{k} \widetilde{H}_{k}(P ; \mathbf{k}) .
$$


Note that $L(P ; G)=(-1)^{r} \widetilde{H}_{r}(P ; \mathbf{k})$, if $P$ is Cohen-Macaulay over $\mathbf{k}$ of rank $r$.

Given finite graded posets $P$ and $Q$ with rank functions $\rho_{P}$ and $\rho_{Q}$, respectively, their Rees product is defined in [8] as $P * Q=\left\{(p, q) \in P \times Q: \rho_{P}(p) \geqslant \rho_{Q}(q)\right\}$, with partial order defined by setting $\left(p_{1}, q_{1}\right) \preceq\left(p_{2}, q_{2}\right)$ if all of the following conditions are satisfied:

- $p_{1} \preceq p_{2}$ holds in $P$,

- $q_{1} \preceq q_{2}$ holds in $Q$ and

- $\rho_{P}\left(p_{2}\right)-\rho_{P}\left(p_{1}\right) \geqslant \rho_{Q}\left(q_{2}\right)-\rho_{Q}\left(q_{1}\right)$.

Equivalently, $\left(p_{1}, q_{1}\right)$ is covered by $\left(p_{2}, q_{2}\right)$ in $P * Q$ if and only if (a) $p_{1}$ is covered by $p_{2}$ in $P$; and (b) either $q_{1}=q_{2}$, or $q_{1}$ is covered by $q_{2}$ in $Q$. We note that the Rees product $P * Q$ is graded with rank function given by $\rho(p, q)=\rho_{P}(p)$ for $(p, q) \in P * Q$, and that if $P$ is a $G$-poset, then so is $P * Q$ with the $G$-action defined by setting $g \cdot(p, q)=(g \cdot p, q)$ for $g \in G$ and $(p, q) \in P * Q$.

A bounded graded $G$-poset $P$, with maximum element $\hat{1}$, is said to be $G$-uniform [17, Section 3] if the following hold:

- the intervals $[x, \hat{1}]$ and $[y, \hat{1}]$ in $P$ are isomorphic for all $x, y \in P$ of the same rank,

- the stabilizers $G_{x}$ and $G_{y}$ are isomorphic for all $x, y \in P$ of the same rank, and

- there is an isomorphism between $[x, \hat{1}]$ and $[y, \hat{1}]$ that intertwines the actions of $G_{x}$ and $G_{y}$, for all $x, y \in P$ of the same rank.

Given a sequence of groups $G=\left(G_{0}, G_{1}, \ldots, G_{n}\right)$, a sequence of posets $\left(P_{0}, P_{1}, \ldots, P_{n}\right)$ is said to be $G$-uniform [17, Section 3] if

- $P_{k}$ is $G_{k}$-uniform of rank $k$ for all $k$,

- $G_{k}$ is isomorphic to the stabilizer $\left(G_{n}\right)_{x}$ for every $x \in P_{n}$ of rank $n-k$ and all $k$, and

- there is an isomorphism between $P_{k}$ and the interval $[x, \hat{1}]$ in $P_{n}$ that intertwines the actions of $G_{k}$ and $\left(G_{n}\right)_{x}$ for every $x \in P_{n}$ of rank $n-k$ and all $k$.

Under these assumptions, Shareshian and Wachs [17, Proposition 3.7] established the isomorphism of $G$-representations

$$
1_{G_{n}} \oplus \bigoplus_{k=0}^{n} W_{k}\left(P_{n} ; G_{n}\right)[k+1]_{t} L\left(\left(P_{n-k} * T_{t, n-k}\right)_{-} ; G_{n-k}\right) \uparrow_{G_{n-k}}^{G_{n}} \cong_{G} 0
$$

for every positive integer $t$, where $W_{k}\left(P_{n} ; G_{n}\right)$ is the number of $G_{n}$-orbits of elements of $P_{n}$ of rank $k$ and $[k+1]_{t}:=1+t+\cdots+t^{k}$.

EXAMPLE 2.1. The Boolean algebra $B_{n}$ consists of all subsets of $[n]$, partially ordered by inclusion. When endowed with the standard $\mathfrak{S}_{n}$-action, it becomes a prototypical example of an $\mathfrak{S}_{n}$-uniform poset. Every element $x \in B_{n}$ of rank $k$ is a set of cardinality $k$; its stabilizer $\left(\mathfrak{S}_{n}\right)_{x}=\left\{w \in \mathfrak{S}_{n}: w x=x\right\}$ is isomorphic to the Young subgroup $\mathfrak{S}_{k} \times \mathfrak{S}_{n-k}$ of $\mathfrak{S}_{n}$, which can be defined as the stalilizer of $[k]$. The sequence $\left(B_{0}, B_{1}, \ldots, B_{n}\right)$ can easily be verified to be $\left(G_{0}, G_{1}, \ldots, G_{n}\right)$-uniform for $G_{k}:=\mathfrak{S}_{k} \times \mathfrak{S}_{n-k}$.

2.2. Permutations, Young tableaux and symmetric functions. Our notation concerning these topics follows mostly that of [15] [25, Chapter 7] [26, Chapter 1]. In particular, the set of standard Young tableaux of shape $\lambda$ is denoted by $\operatorname{SYT}(\lambda)$, the descent set $\{i \in[n-1]: w(i)>w(i+1)\}$ of a permutation $w \in \mathfrak{S}_{n}$ is denoted by $\operatorname{Des}(w)$ and that of a tableau $Q \in \operatorname{SYT}(\lambda)$, consisting of those entries $i$ for which $i+1$ appears in $Q$ in a lower row than $i$, is denoted by $\operatorname{Des}(Q)$. We recall that the Robinson-Schensted correspondence is a bijection from the symmetric group $\mathfrak{S}_{n}$ to 
the set of pairs $(\mathcal{P}, Q)$ of standard Young tableaux of the same shape and size $n$. This correspondence has the property [25, Lemma 7.23.1] that $\operatorname{Des}(w)=\operatorname{Des}(Q(w))$ and $\operatorname{Des}\left(w^{-1}\right)=\operatorname{Des}(\mathcal{P}(w))$, where $(\mathcal{P}(w), Q(w))$ is the pair of tableaux associated to $w \in \mathfrak{S}_{n}$.

We will consider symmetric functions in the indeterminates $\mathbf{x}=\left(x_{1}, x_{2}, \ldots\right)$ over the complex field $\mathbb{C}$. We denote by $E(\mathbf{x} ; z):=\sum_{n \geqslant 0} e_{n}(\mathbf{x}) z^{n}$ and $H(\mathbf{x} ; z):=$ $\sum_{n \geqslant 0} h_{n}(\mathbf{x}) z^{n}$ the generating functions for the elementary and complete homogeneous symmetric functions, respectively, and recall the identity $E(\mathbf{x} ; z) H(\mathbf{x} ;-z)=1$. The (Frobenius) characteristic map, a $\mathbb{C}$-linear isomorphism of fundamental importance from the space of virtual $\mathfrak{S}_{n}$-representations to that of homogeneous symmetric functions of degree $n$, will be denoted by ch. This map sends the irreducible $\mathfrak{S}_{n^{-}}$ representation corresponding to $\lambda \vdash n$ to the Schur function $s_{\lambda}(\mathbf{x})$ associated to $\lambda$ and thus it sends non-virtual $\mathfrak{S}_{n}$-representations to Schur-positive symmetric functions. The fundamental quasisymmetric function associated to $S \subseteq[n-1]$ is defined as

$$
F_{n, S}(\mathbf{x})=\sum_{\substack{1 \leqslant i_{1} \leqslant i_{2} \leqslant \cdots \leqslant i_{n} \\ j \in S \Rightarrow i_{j}<i_{j+1}}} x_{i_{1}} x_{i_{2}} \cdots x_{i_{n}} .
$$

The following well known expansion [25, Theorem 7.19.7]

$$
s_{\lambda}(\mathbf{x})=\sum_{Q \in \operatorname{SYT}(\lambda)} F_{n, \operatorname{Des}(Q)}(\mathbf{x})
$$

will be used in Section 4 .

For the applications given there, we need the analogues of these concepts in the representation theory of the hyperoctahedral group of signed permutations of the set $[n]$, denoted here by $\mathcal{B}_{n}$. We will keep this discussion rather brief and refer to $[1$, Section 2] for more information.

A bipartition of a positive integer $n$, written $(\lambda, \mu) \vdash n$, is any pair $(\lambda, \mu)$ of integer partitions of total sum $n$. A standard Young bitableau of shape $(\lambda, \mu) \vdash n$ is any pair $Q=\left(Q^{+}, Q^{-}\right)$of Young tableaux such that $Q^{+}$has shape $\lambda, Q^{-}$has shape $\mu$ and every element of $[n]$ appears exactly once as an entry of $Q^{+}$or $Q^{-}$. The tableaux $Q^{+}$ and $Q^{-}$are called the parts of $Q$ and the number $n$ is its size. The Robinson-Schensted correspondence of type $B$, as described in [21, Section 6] (see also [1, Section 5]), is a bijection from the group $\mathcal{B}_{n}$ to the set of pairs $(\mathcal{P}, Q)$ of standard Young bitableaux of the same shape and size $n$.

The (Frobenius) characteristic map for the hyperoctahedral group, denoted by $\mathrm{ch}_{\mathcal{B}}$, is a $\mathbb{C}$-linear isomorphism from the space of virtual $\mathcal{B}_{n}$-representations to that of homogeneous symmetric functions of degree $n$ in the sets of indeterminates $\mathbf{x}=\left(x_{1}, x_{2}, \ldots\right)$ and $\mathbf{y}=\left(y_{1}, y_{2}, \ldots\right)$ separately; see, for instance, [27, Section 5]. The map $\operatorname{ch}_{\mathcal{B}}$ sends the irreducible $\mathcal{B}_{n}$-representation corresponding to $(\lambda, \mu) \vdash n$ to the function $s_{\lambda}(\mathbf{x}) s_{\mu}(\mathbf{y})$ and thus it sends non-virtual $\mathcal{B}_{n}$-representations to Schur-positive functions, meaning nonnegative integer linear combinations of the functions $s_{\lambda}(\mathbf{x}) s_{\mu}(\mathbf{y})$. The following basic properties of $\mathrm{ch}_{\mathcal{B}}$ will be useful in Section 4:

- $\operatorname{ch}_{\mathcal{B}}\left(1_{\mathcal{B}_{n}}\right)=h_{n}(\mathbf{x})$, where $1_{\mathcal{B}_{n}}$ is the trivial $\mathcal{B}_{n}$-representation,

- $\operatorname{ch}_{\mathcal{B}}\left(\sigma \otimes \tau \uparrow_{\mathcal{B}_{k} \times \mathcal{B}_{n-k}}^{\mathcal{B}_{n}}\right)=\operatorname{ch}_{\mathcal{B}}(\sigma) \cdot \operatorname{ch}_{\mathcal{B}}(\tau)$ for all representations $\sigma$ and $\tau$ of $\mathcal{B}_{k}$ and $\mathcal{B}_{n-k}$, respectively, where $\mathcal{B}_{k} \times \mathcal{B}_{n-k}$ is the Young subgroup of $\mathcal{B}_{n}$ consisting of all signed permutations which preserve the set $\{ \pm 1, \pm 2, \ldots, \pm k\}$,

- $\operatorname{ch}_{\mathcal{B}}\left(\uparrow_{\mathfrak{S}_{n}}^{\mathcal{B}_{n}} \rho\right)=\operatorname{ch}(\rho)(\mathbf{x}, \mathbf{y})$ for every $\mathfrak{S}_{n}$-representation $\rho$, where $\mathfrak{S}_{n} \subset \mathcal{B}_{n}$ is the standard embedding.

We denote by $E(\mathbf{x}, \mathbf{y} ; z):=\sum_{n \geqslant 0} e_{n}(\mathbf{x}, \mathbf{y}) z^{n}$ and $H(\mathbf{x}, \mathbf{y} ; z):=\sum_{n \geqslant 0} h_{n}(\mathbf{x}, \mathbf{y}) z^{n}$ the generating function for the elementary and complete homogeneous, respectively, 
symmetric functions in the variables $(\mathbf{x}, \mathbf{y})=\left(x_{1}, x_{2}, \ldots, y_{1}, y_{2}, \ldots\right)$ and note that $E(\mathbf{x}, \mathbf{y} ; z)=E(\mathbf{x} ; z) E(\mathbf{y} ; z)$, since $e_{n}(\mathbf{x}, \mathbf{y})=\sum_{k=0}^{n} e_{k}(\mathbf{x}) e_{n-k}(\mathbf{y})$, and similarly that $H(\mathbf{x}, \mathbf{y} ; z)=H(\mathbf{x} ; z) H(\mathbf{y} ; z)$.

The signed descent set [1, Section 2] [13] of $w \in \mathcal{B}_{n}$, denoted $\operatorname{sDes}(w)$, records the positions of the increasing (in absolute value) runs of constant sign in the sequence $(w(1), w(2), \ldots, w(n))$. Formally, we may define $\operatorname{sDes}(w)$ as the pair $(\operatorname{Des}(w), \varepsilon)$, where $\varepsilon=\left(\varepsilon_{1}, \varepsilon_{2}, \ldots, \varepsilon_{n}\right) \in\{-,+\}^{n}$ is the sign vector with $i$ th coordinate equal to the sign of $w(i)$ and $\operatorname{Des}(w)$ consists of the indices $i \in[n-1]$ for which either $\varepsilon_{i}=+$ and $\varepsilon_{i+1}=-$, or else $\varepsilon_{i}=\varepsilon_{i+1}$ and $|w(i)|>|w(i+1)|$ (this definition is slightly different from, but equivalent to, the ones given in $[1,13])$. The fundamental quasisymmetric function associated to $w$, introduced by Poirier [13] in a more general setting, is defined as

$$
F_{\mathrm{sDes}(w)}(\mathbf{x}, \mathbf{y})=\sum_{\substack{i_{1} \leqslant i_{2} \leqslant \cdots \leqslant i_{n} \\ j \in \operatorname{Des}(w) \Rightarrow i_{j}<i_{j+1}}} z_{i_{1}} z_{i_{2}} \cdots z_{i_{n}},
$$

where $z_{i_{j}}=x_{i_{j}}$ if $\varepsilon_{j}=+$, and $z_{i_{j}}=y_{i_{j}}$ if $\varepsilon_{j}=-$. Given a standard Young bitableau $Q$ of size $n$, one defines the signed descent set $\operatorname{sDes}(Q)$ as the pair $(\operatorname{Des}(Q), \varepsilon)$, where $\varepsilon=\left(\varepsilon_{1}, \varepsilon_{2}, \ldots, \varepsilon_{n}\right) \in\{-,+\}^{n}$ is the sign vector with $i$ th coordinate equal to the sign of the part of $Q$ in which $i$ appears and $\operatorname{Des}(Q)$ is the set of indices $i \in[n-1]$ for which either $\varepsilon_{i}=+$ and $\varepsilon_{i+1}=-$, or else $\varepsilon_{i}=\varepsilon_{i+1}$ and $i+1$ appears in $Q$ in a lower row than $i$. The function $F_{s \operatorname{Des}(Q)}(\mathbf{x}, \mathbf{y})$ is then defined by the right-hand side of Equation (16), with $w$ replaced by $Q$; see [1, Section 2]. The analogue

$$
s_{\lambda}(\mathbf{x}) s_{\mu}(\mathbf{y})=\sum_{Q \in \operatorname{SYT}(\lambda, \mu)} F_{s \operatorname{Des}(Q)}(\mathbf{x}, \mathbf{y})
$$

of the expansion (15) holds ([1, Proposition 4.2]) and the Robinson-Schensted correspondence of type $B$ has the properties that $\operatorname{sDes}(w)=\operatorname{sDes}\left(Q^{B}(w)\right)$ and $\operatorname{sDes}\left(w^{-1}\right)=\operatorname{sDes}\left(\mathcal{P}^{B}(w)\right)$, where $\left(\mathcal{P}^{B}(w), Q^{B}(w)\right)$ is the pair of bitableaux associated to $w \in \mathcal{B}_{n}$; see $[1$, Proposition 5.1].

\section{Proof of Theorem 1.1}

This section proves Theorem 1.1 using only the definition of Rees product and the defining equation (2) of the representations $\beta_{P}(S)$. For $S=\left\{s_{1}, s_{2}, \ldots, s_{k}\right\} \subseteq[n]$ with $s_{1}<s_{2}<\cdots<s_{k}$ we set

$$
\begin{aligned}
\varphi_{t}(S) & :=\left[s_{1}+1\right]_{t}\left[s_{2}-s_{1}+1\right]_{t} \cdots\left[s_{k}-s_{k-1}+1\right]_{t} \\
\psi_{t}(S) & :=\left[s_{1}\right]_{t}\left[s_{2}-s_{1}+1\right]_{t} \cdots\left[s_{k}-s_{k-1}+1\right]_{t}
\end{aligned}
$$

where, as mentioned already, $[m]_{t}=1+t+\cdots+t^{m-1}$ for positive integers $m$.

Lemma 3.1. Let $G$ be a finite group, $P$ be a finite bounded graded $G$-poset of rank $n+1$, as in Theorem 1.1, and $Q, R$ be the posets defined by $\bar{Q}=\left(P^{-} * T_{t, n}\right)_{-}$and $\bar{R}=\bar{P} * T_{t, n-1}$. Then,

$$
\begin{aligned}
& \alpha_{Q}(S) \cong_{G} \varphi_{t}(S) \alpha_{P}(S) \\
& \alpha_{R}(S) \cong_{G} \psi_{t}(S) \alpha_{P}(S)
\end{aligned}
$$

for all positive integers $t$ and $S \subseteq[n]$.

Proof. Let $S=\left\{s_{1}, s_{2}, \ldots, s_{k}\right\} \subseteq[n]$ with $s_{1}<s_{2}<\cdots<s_{k}$ and let $\rho: T_{t, n} \rightarrow \mathbb{N}$ be the rank function of $T_{t, n}$. By the definition of Rees product, the maximal chains in $Q_{S}$ have the form

$$
\hat{0} \prec\left(p_{1}, \tau_{1}\right) \prec\left(p_{2}, \tau_{2}\right) \prec \cdots \prec\left(p_{k}, \tau_{k}\right) \prec \hat{1}
$$


where $\hat{0} \prec p_{1} \prec p_{2} \prec \cdots \prec p_{k} \prec \hat{1}$ is a maximal chain in $P_{S}$ and $\tau_{1} \preceq \tau_{2} \preceq \cdots \preceq \tau_{k}$ is a multichain in $T_{t, n}$ such that

- $0 \leqslant \rho\left(\tau_{1}\right) \leqslant s_{1}$ and

- $\rho\left(\tau_{j}\right)-\rho\left(\tau_{j-1}\right) \leqslant s_{j}-s_{j-1}$ for $2 \leqslant j \leqslant k$.

Let $m_{t}(S)$ be the number of these multichains. Since the elements of $G$ act on (18) by fixing the $\tau_{j}$ and acting on the corresponding maximal chain of $P_{S}$, we have $\alpha_{Q}(S) \cong_{G}$ $m_{t}(S) \alpha_{P}(S)$. To choose such a multichain $\tau_{1} \preceq \tau_{2} \preceq \cdots \preceq \tau_{k}$, we need to specify the sequence $i_{1} \leqslant i_{2} \leqslant \cdots \leqslant i_{k}$ of ranks of its elements so that $i_{j}-i_{j-1} \leqslant s_{j}-s_{j-1}$ for $1 \leqslant j \leqslant k$, where $i_{0}:=s_{0}:=0$, and choose its maximum element $\tau_{k}$ in $t^{i_{k}}$ possible ways. Summing over all such sequences, we get

$$
m_{t}(S)=\sum_{\left(i_{1}, i_{2}, \ldots, i_{k}\right)} t^{i_{k}}=\sum_{0 \leqslant a_{j} \leqslant s_{j}-s_{j-1}} t^{a_{1}+a_{2}+\cdots+a_{k}}=\varphi_{t}(S)
$$

and the result for $\alpha_{Q}(S)$ follows. The same argument applies to $\alpha_{R}(S)$; one simply has to switch the condition for the rank of $\tau_{1}$ to $0 \leqslant \rho\left(\tau_{1}\right) \leqslant s_{1}-1$.

The proof of the following technical lemma will be given after that of Theorem 1.1.

LEMma 3.2. For every $S \subseteq[n]$ we have

$$
\sum_{S \subseteq T \subseteq[n]}(-1)^{n-|T|} \varphi_{t}(T)= \begin{cases}0, & \text { if }[n] \backslash S \text { is not stable } \\ t^{r}(1+t)^{n-2 r}, & \text { if }[n] \backslash S \text { is stable and } n \in S, \\ t^{r}(1+t)^{n+1-2 r}, & \text { if }[n] \backslash S \text { is stable and } n \notin S\end{cases}
$$

and

$$
\sum_{S \subseteq T \subseteq[n]}(-1)^{n-|T|} \psi_{t}(T)= \begin{cases}0, & \text { if } 1 \notin S, \\ 0, & \text { if }[n] \backslash S \text { is not stable, } \\ t^{r}(1+t)^{n-1-2 r}, & \text { if }[n] \backslash S \text { is stable and } 1, n \in S, \\ t^{r}(1+t)^{n-2 r}, & \text { if }[n] \backslash S \text { is stable, } 1 \in S \text { and } n \notin S,\end{cases}
$$

where $r=n-|S|$.

Proof of Theorem 1.1. Using Equations (2) and (3), as well as Lemma 3.1, we compute that

$$
\begin{aligned}
\beta_{Q}([n]) & =\sum_{T \subseteq[n]}(-1)^{n-|T|} \alpha_{Q}(T) \cong_{G} \sum_{T \subseteq[n]}(-1)^{n-|T|} \varphi_{t}(T) \alpha_{P}(T) \\
& =\sum_{T \subseteq[n]}(-1)^{n-|T|} \varphi_{t}(T) \sum_{S \subseteq T} \beta_{P}(S) \\
& =\sum_{S \subseteq[n]} \beta_{P}(S) \sum_{S \subseteq T \subseteq[n]}(-1)^{n-|T|} \varphi_{t}(T)
\end{aligned}
$$

and find similarly that

$$
\beta_{R}([n]) \cong_{G} \sum_{S \subseteq[n]} \beta_{P}(S) \sum_{S \subseteq T \subseteq[n]}(-1)^{n-|T|} \psi_{t}(T) .
$$

The proof follows from these formulas and Lemma 3.2. For the last statement of the theorem one has to note that, as a consequence of [8, Corollary 2], if $P$ is CohenMacaulay over $\mathbf{k}$, then so are the Rees products $P^{-} * T_{t, n}$ and $\bar{P} * T_{t, n-1}$.

Proof of Lemma 3.2. Let us denote by $\chi_{t}(S)$ the left-hand side of (19) and write $S=\left\{s_{1}, s_{2}, \ldots, s_{k}\right\}$, with $1 \leqslant s_{1}<s_{2}<\cdots<s_{k} \leqslant n$. By definition, we have

$$
\chi_{t}(S)=\chi_{t}\left(s_{1}\right) \chi_{t}\left(s_{2}-s_{1}\right) \cdots \chi_{t}\left(s_{k}-s_{k-1}\right) \omega_{t}\left(n-s_{k}\right),
$$


where

$$
\begin{aligned}
\chi_{t}(n) & :=\sum_{n \in T \subseteq[n]}(-1)^{n-|T|} \varphi_{t}(T) \\
\omega_{t}(n) & :=\sum_{T \subseteq[n]}(-1)^{n-|T|} \varphi_{t}(T)
\end{aligned}
$$

for $n \geqslant 1$ and $\omega_{t}(0):=1$. We claim that

$$
\chi_{t}(n)=\left\{\begin{array}{ll}
1+t, & \text { if } n=1, \\
t, & \text { if } n=2, \\
0, & \text { if } n \geqslant 3
\end{array} \quad \text { and } \quad \omega_{t}(n)= \begin{cases}1, & \text { if } n=0 \\
t, & \text { if } n=1, \\
0, & \text { if } n \geqslant 2\end{cases}\right.
$$

Equation (19) is a direct consequence of (21) and this claim. To verify the claim, note that the defining equation for $\chi_{t}(n)$ can be rewritten as

$$
\chi_{t}(n)=\sum_{\left(a_{1}, a_{2}, \ldots, a_{k}\right) \models n}(-1)^{n-k}\left[a_{1}+1\right]_{t}\left[a_{2}+1\right]_{t} \cdots\left[a_{k}+1\right]_{t},
$$

where the sum ranges over all sequences (compositions) $\left(a_{1}, a_{2}, \ldots, a_{k}\right)$ of positive integers summing to $n$. We leave it as a simple combinatorial exercise for the interested reader to show (for instance, by standard generating function methods) that $\chi_{t}(n)=0$ for $n \geqslant 3$. The claim follows from this fact and the obvious recurrence $\omega_{t}(n)=$ $\chi_{t}(n)-\omega_{t}(n-1)$, valid for $n \geqslant 1$.

Finally, note that Equation (20) is equivalent to (19) in the case $1 \in S$. Otherwise, the terms in the left-hand side can be partitioned into pairs of terms, corresponding to pairs $\{T, T \cup\{1\}\}$ of subsets with $1 \notin T$, canceling each other. This shows that the left-hand side vanishes.

\section{Symmetric FunCtion identities}

This section derives Equations (6)-(11) from Theorem 1.1 (Corollaries 4.1, 4.4 and 4.7) and interprets combinatorially the Schur-positive symmetric functions which appear there. We first explain why Gessel's identities are special cases of this theorem. The set of ascents of a permutation $w \in \mathfrak{S}_{n}$ is defined as $\operatorname{Asc}(w):=[n-1] \backslash \operatorname{Des}(w)$ and, similarly, we have $\operatorname{Asc}(\mathcal{P}):=[n-1] \backslash \operatorname{Des}(\mathcal{P})$ for every standard Young tableau $\mathcal{P}$ of size $n$. Let us recall the fact (used in the following proof) that the reduced homology groups of a poset with a minimum or maximum element vanish.

COROLlary 4.1. Equations (6) and (7) are valid for the functions

$$
\xi_{n, k}(\mathbf{x})=\sum_{\lambda \vdash n} c_{\lambda, k} \cdot s_{\lambda}(\mathbf{x})=\sum_{w} F_{n, \operatorname{Des}(w)}(\mathbf{x})
$$

and

$$
\gamma_{n, k}(\mathbf{x})=\sum_{\lambda \vdash n} d_{\lambda, k} \cdot s_{\lambda}(\mathbf{x})=\sum_{w} F_{n, \operatorname{Des}(w)}(\mathbf{x}),
$$

where $c_{\lambda, k}$ (respectively, $d_{\lambda, k}$ ) stands for the number of tableaux $\mathcal{P} \in \operatorname{SYT}(\lambda)$ for which $\operatorname{Asc}(\mathcal{P}) \in \operatorname{Stab}([2, n-2])($ respectively, $\operatorname{Asc}(\mathcal{P}) \in \operatorname{Stab}([n-2]))$ has $k$ elements and, similarly, $w \in \mathfrak{S}_{n}$ runs through all permutations for which $\operatorname{Asc}\left(w^{-1}\right) \in \operatorname{Stab}([2, n-2])$ (respectively, $\left.\operatorname{Asc}\left(w^{-1}\right) \in \operatorname{Stab}([n-2])\right)$ has $k$ elements.

Proof. We will apply Theorem 1.1 when $P^{-}$is the Boolean lattice $B_{n}$, considered as an $\mathfrak{S}_{n}$-poset as in Example 2.1. On the one hand, we have the equality

$$
1+\sum_{n \geqslant 2} \operatorname{ch}\left(\widetilde{H}_{n-1}\left(\left(B_{n} \backslash\{\varnothing\}\right) * T_{t, n-1} ; \mathbb{C}\right)\right) z^{n}=\frac{1-t}{E(\mathbf{x} ; t z)-t E(\mathbf{x} ; z)}
$$


which, although not explicitly stated in [17], follows as in the proof of its special case $t=1$ [17, Corollary 5.2]. On the other hand, since $B_{n}$ has a maximum element, the second summand in the right-hand side of Equation (5) vanishes and hence this equation gives

$$
\begin{aligned}
\operatorname{ch}\left(\widetilde { H } _ { n - 1 } \left(\left(B_{n} \backslash\{\varnothing\}\right)\right.\right. & \left.\left.* T_{t, n-1} ; \mathbb{C}\right)\right) \\
& =\sum_{S \in \operatorname{Stab}([2, n-2])} \operatorname{ch}\left(\beta_{B_{n}}([n-1] \backslash S)\right) t^{|S|+1}(1+t)^{n-2-2|S|}
\end{aligned}
$$

for $n \geqslant 2$. The representations $\beta_{B_{n}}(S)$ for $S \subseteq[n-1]$ are known to satisfy (see, for instance, [21, Theorem 4.3])

$$
\operatorname{ch}\left(\beta_{B_{n}}(S)\right)=\sum_{\lambda \vdash n} c_{\lambda, S} \cdot s_{\lambda}(\mathbf{x}),
$$

where $c_{\lambda, S}$ is the number of standard Young tableaux of shape $\lambda$ and descent set equal to $S$. Combining the previous three equalities yields the first equality in Equation (22). The second equality follows from the first by expanding $s_{\lambda}(\mathbf{x})$ according to Equation (15) to get

$$
\xi_{n, k}(\mathbf{x})=\sum_{\lambda \vdash n} \sum_{\mathcal{P}} \sum_{Q \in \mathrm{SYT}(\lambda)} F_{n, \operatorname{Des}(Q)}(\mathbf{x})
$$

where, in the inner sum, $\mathcal{P}$ runs through all tableaux in $\operatorname{SYT}(\lambda)$ for which $\operatorname{Asc}(\mathcal{P}) \in$ $\mathcal{P}_{\text {Stab }}([2, n-2])$ has $k$ elements, and then using the Robinson-Schensted correspondence and its standard properties $\operatorname{Des}(w)=\operatorname{Des}(Q(w))$ and $\operatorname{Des}\left(w^{-1}\right)=\operatorname{Des}(\mathcal{P}(w))$ to replace the summations with one running over elements of $\mathfrak{S}_{n}$, as in the statement of the corollary.

The proof of (23) is entirely similar; one has to use Equation (4) instead of (5), as well as the equality

$$
1+\sum_{n \geqslant 1} \operatorname{ch}\left(\widetilde{H}_{n-1}\left(\left(B_{n} * T_{t, n}\right)_{-} ; \mathbb{C}\right)\right) z^{n}=\frac{(1-t) E(\mathbf{x} ; t z)}{E(\mathbf{x} ; t z)-t E(\mathbf{x} ; z)} .
$$

The latter follows from the proof of Equation (3.3) in [17, pp. 15-16], where the left-hand side is equal to $-F_{t}(-z)$, in the notation used in that proof.

ExAmPLE 4.2. The coefficient of $z^{4}$ in the left-hand sides of Equations (6) and (7) equals

- $e_{4}(\mathbf{x})\left(t+t^{2}+t^{3}\right)+e_{2}(\mathbf{x})^{2} t^{2}$, and

- $e_{4}(\mathbf{x})\left(t+t^{2}+t^{3}+t^{4}\right)+e_{1}(\mathbf{x}) e_{3}(\mathbf{x})\left(t^{2}+t^{3}\right)+e_{2}(\mathbf{x})^{2}\left(t^{2}+t^{3}\right)$,

respectively. These expressions may be rewritten as

- $s_{(1,1,1,1)}(\mathbf{x}) t(1+t)^{2}+s_{(2,1,1)}(\mathbf{x}) t^{2}+s_{(2,2)}(\mathbf{x}) t^{2}$, and

- $s_{(1,1,1,1)}(\mathbf{x}) t(1+t)^{3}+2 s_{(2,1,1)}(\mathbf{x}) t^{2}(1+t)+s_{(2,2)}(\mathbf{x}) t^{2}(1+t)$,

respectively. Hence, $\xi_{4,0}(\mathbf{x})=s_{(1,1,1,1)}(\mathbf{x}), \xi_{4,1}(\mathbf{x})=s_{(2,1,1)}(\mathbf{x})+s_{(2,2)}(\mathbf{x}), \gamma_{4,0}(\mathbf{x})=$ $s_{(1,1,1,1)}(\mathbf{x})$ and $\gamma_{4,1}(\mathbf{x})=2 s_{(2,1,1)}(\mathbf{x})+s_{(2,2)}(\mathbf{x})$. We leave it to the reader to verify that these formulas agree with Corollary 4.1 .

We now focus on the identities (8)-(11). We will apply Theorem 1.1 to the collection $s B_{n}$ of all subsets of $\{1,2, \ldots, n\} \cup\{-1,-2, \ldots,-n\}$ which do not contain $\{i,-i\}$ for any index $i$, partially ordered by inclusion. This signed analogue of the Boolean algebra $B_{n}$ is a graded poset of rank $n$, having the empty set as its minimum element, on which the hyperoctahedral group $\mathcal{B}_{n}$ acts in the obvious way [21, Section 6], turning it into a $\mathcal{B}_{n}$-poset. It is isomorphic to the poset of faces (including the empty one) 
of the boundary complex of the $n$-dimensional cross-polytope and hence it is CohenMacaulay over $\mathbb{Z}$ and any field. The left-hand sides of Equations (4) and (5) for $P^{-}=s B_{n}$ will be computed using the methods of [17].

Consider the $n$-element chain $C_{n}=\{0,1, \ldots, n-1\}$, with the usual total order. Following [17], we denote by $I_{j}\left(B_{n}\right)$ the order ideal of elements of $\left(B_{n} \backslash\{\varnothing\}\right) * C_{n}$ which are strictly less than $([n], j)$. Then $I_{j}\left(B_{n}\right)$ is an $\mathfrak{S}_{n}$-poset for every $j \in C_{n}$ and one of the main results of [17] (see [17, p. 21] [19, Equation (2.5)]) states that

$$
1+\sum_{n \geqslant 1} z^{n} \sum_{j=0}^{n-1} t^{j} \operatorname{ch}\left(\widetilde{H}_{n-2}\left(I_{j}\left(B_{n}\right) ; \mathbb{C}\right)\right)=\frac{(1-t) E(\mathbf{x} ; z)}{E(\mathbf{x} ; t z)-t E(\mathbf{x} ; z)} .
$$

Proposition 4.3. For the $\mathcal{B}_{n}$-poset $s B_{n}$ we have

$$
\begin{aligned}
1+\sum_{n \geqslant 1} \operatorname{ch}_{\mathcal{B}}\left(\widetilde{H}_{n-1}\left(\left(s B_{n} \backslash\{\varnothing\}\right) * T_{t, n-1} ; \mathbb{C}\right)\right) z^{n} \\
=\frac{(1-t) E(\mathbf{y} ; z)}{E(\mathbf{x} ; t z) E(\mathbf{y} ; t z)-t E(\mathbf{x} ; z) E(\mathbf{y} ; z)} .
\end{aligned}
$$

Proof. Following the reasoning in the proof of [17, Corollary 5.2], we apply (12) to the Cohen-Macaulay $\mathcal{B}_{n}$-poset obtained from $\left(s B_{n} \backslash\{\varnothing\}\right) * T_{t, n-1}$ by adding a minimum and a maximum element. For $0 \leqslant j<k \leqslant n$, there are exactly $t^{j} \mathcal{B}_{n}$-orbits of elements $x$ of rank $k$ in this poset with second coordinate of rank $j$ in $T_{t, n-1}$ and for each one of these, the open interval $(\hat{0}, x)$ is isomorphic to $I_{j}\left(B_{k}\right)$ and the stabilizer of $x$ is isomorphic to $\mathfrak{S}_{k} \times \mathcal{B}_{n-k}$. We conclude that

$$
\begin{aligned}
& \widetilde{H}_{n-1}\left(\left(s B_{n} \backslash\{\varnothing\}\right) * T_{t, n-1} ; \mathbb{C}\right) \\
& \cong \mathcal{B}_{n} \bigoplus_{k=0}^{n}(-1)^{n-k} \bigoplus_{j=0}^{k-1} t^{j}\left(\widetilde{H}_{k-2}\left(I_{j}\left(B_{k}\right) ; \mathbb{C}\right) \otimes 1_{\mathcal{B}_{n-k}}\right) \uparrow_{\mathfrak{S}_{k} \times \mathcal{B}_{n-k}}^{\mathcal{B}_{n}} .
\end{aligned}
$$

Applying the map $\operatorname{ch}_{\mathcal{B}}$ and using the transitivity $\uparrow_{\mathfrak{S}_{k} \times \mathcal{B}_{n-k}}^{\mathcal{B}_{n}} \cong \mathcal{B}_{n} \uparrow \mathfrak{\mathcal { S }}_{k} \times \mathcal{B}_{n-k} \quad \uparrow \mathcal{B}_{n-k} \mathcal{B}_{n} \times \mathcal{B}_{n-k}$ of induction and properties of $\operatorname{ch}_{\mathcal{B}}$ discussed in Section 2, the right-hand side becomes

$$
\sum_{k=0}^{n}(-1)^{n-k} \sum_{j=0}^{k-1} t^{j} \operatorname{ch}\left(\widetilde{H}_{k-2}\left(I_{j}\left(B_{k}\right) ; \mathbb{C}\right)\right)(\mathbf{x}, \mathbf{y}) \cdot h_{n-k}(\mathbf{x}) .
$$

Thus, the left-hand side of Equation (25) is equal to

$$
H(\mathbf{x} ;-z) \cdot\left(1+\sum_{n \geqslant 1} z^{n} \sum_{j=0}^{n-1} t^{j} \operatorname{ch}\left(\widetilde{H}_{n-2}\left(I_{j}\left(B_{n}\right) ; \mathbb{C}\right)\right)(\mathbf{x}, \mathbf{y})\right)
$$

and the result follows from Equation (24) and the identities $E(\mathbf{x} ; z) H(\mathbf{x} ;-z)=1$ and $E(\mathbf{x}, \mathbf{y} ; z)=E(\mathbf{x} ; z) E(\mathbf{y} ; z)$.

Recall the definition of the sets $\operatorname{Des}(w)$ and $\operatorname{Des}(\mathcal{P})$ for a signed permutation $w \in \mathcal{B}_{n}$ and standard Young bitableau $\mathcal{P}$ of size $n$, respectively, from Section 2. Following $[21$, Section 6], we define the type $B$ descent set of $\mathcal{P}=\left(\mathcal{P}^{+}, \mathcal{P}^{-}\right)$as $\operatorname{Des}_{B}(\mathcal{P})=\operatorname{Des}(\mathcal{P}) \cup$ $\{n\}$, if $n$ appears in $\mathcal{P}^{+}$, and $\operatorname{Des}_{B}(\mathcal{P})=\operatorname{Des}(\mathcal{P})$ otherwise. The complement of $\operatorname{Des}_{B}(\mathcal{P})$ in the set $[n]$ is called the type $B$ ascent set of $\mathcal{P}$ and is denoted by $\operatorname{Asc}_{B}(\mathcal{P})$. Similarly, we define the type $B$ descent set of $w \in \mathcal{B}_{n}$ as $\operatorname{Des}_{B}(w)=\operatorname{Des}(w) \cup\{n\}$, if $w(n)$ is positive, and $\operatorname{Des}_{B}(w)=\operatorname{Des}(w)$ otherwise. The complement of $\operatorname{Des}_{B}(w)$ in the set $[n]$ is called the type $B$ ascent set of $w$ and is denoted by $\operatorname{Asc}_{B}(w)$. The sets $\operatorname{Des}_{B}(w)$ and $\operatorname{Des}_{B}(\mathcal{P})$ depend only on the signed descent sets $\operatorname{sDes}_{B}(w)$ and $\operatorname{sDes}_{B}(\mathcal{P})$, respectively, and [1, Proposition 5.1], mentioned at the end of Section 2, 
implies that $\operatorname{Des}_{B}(w)=\operatorname{Des}_{B}\left(Q^{B}(w)\right)$ and $\operatorname{Des}_{B}\left(w^{-1}\right)=\operatorname{Des}_{B}\left(\mathcal{P}^{B}(w)\right)$ for every $w \in \mathcal{B}_{n}$.

COROLlary 4.4. Equations (8) and (9) are valid for the functions

$$
\xi_{n, k}^{+}(\mathbf{x}, \mathbf{y})=\sum_{(\lambda, \mu) \vdash n} c_{(\lambda, \mu), k}^{+} \cdot s_{\lambda}(\mathbf{x}) s_{\mu}(\mathbf{y})=\sum_{w} F_{\mathrm{sDes}(w)}(\mathbf{x}, \mathbf{y})
$$

and

$$
\xi_{n, k}^{-}(\mathbf{x}, \mathbf{y})=\sum_{(\lambda, \mu) \vdash n} c_{(\lambda, \mu), k}^{-} \cdot s_{\lambda}(\mathbf{x}) s_{\mu}(\mathbf{y})=\sum_{w} F_{\mathrm{sDes}(w)}(\mathbf{x}, \mathbf{y}),
$$

where $c_{(\lambda, \mu), k}^{+}\left(\right.$respectively, $\left.c_{(\lambda, \mu), k}^{-}\right)$stands for the number of bitableaux $\mathcal{P} \in \operatorname{SYT}(\lambda, \mu)$ for which $\operatorname{Asc}_{B}(\mathcal{P}) \in \operatorname{Stab}([2, n])$ has $k$ elements and contains (respectively, does not contain) $n$ and where, similarly, $w \in \mathcal{B}_{n}$ runs through all signed permutations for which $\operatorname{Asc}_{B}\left(w^{-1}\right) \in \operatorname{Stab}([2, n])$ has $k$ elements and contains (respectively, does not contain) $n$.

Proof. We apply the second part of Theorem 1.1 for $P^{-}=s B_{n}$, thought of as a $\mathcal{B}_{n}$-poset. The representations $\beta_{P}(S)$ for $S \subseteq[n]$ were computed in this case in [21, Theorem 6.4], which implies that

$$
\operatorname{ch}_{\mathcal{B}}\left(\beta_{B_{n}}(S)\right)=\sum_{(\lambda, \mu) \vdash n} c_{(\lambda, \mu), S} \cdot s_{\lambda}(\mathbf{y}) s_{\mu}(\mathbf{x})
$$

for $S \subseteq[n]$, where $c_{(\lambda, \mu), S}$ is the number of standard Young bitableaux $\mathcal{P}$ of shape $(\lambda, \mu)$ such that $\operatorname{Des}_{B}(\mathcal{P})=S$. Switching the roles of $\mathbf{x}$ and $\mathbf{y}$ and combining this result with the second part of Theorem 1.1 and Proposition 4.3 we get

$$
\begin{aligned}
& \frac{(1-t) E(\mathbf{x} ; z)}{E(\mathbf{x} ; t z) E(\mathbf{y} ; t z)-t E(\mathbf{x} ; z) E(\mathbf{y} ; z)}=\sum_{n \geqslant 0} z^{n} \sum_{k=0}^{\lfloor n / 2\rfloor} \xi_{n, k}^{+}(\mathbf{x}, \mathbf{y}) t^{k}(1+t)^{n-2 k} \\
&+\sum_{n \geqslant 1} z^{n} \sum_{k=0}^{\lfloor(n-1) / 2\rfloor} \xi_{n, k}^{-}(\mathbf{x}, \mathbf{y}) t^{k}(1+t)^{n-1-2 k}
\end{aligned}
$$

where the $\xi_{n, k}^{ \pm}(\mathbf{x}, \mathbf{y})$ are given by the first equalities in (26) and (27). We now note that the left-hand side of Equation (28) is equal to the sum of the left-hand sides, say $\Xi^{+}(\mathbf{x}, \mathbf{y}, t ; z)$ and $\Xi^{-}(\mathbf{x}, \mathbf{y}, t ; z)$, of Equations (8) and (9). Since, as one can readily verify, $\Xi^{+}(\mathbf{x}, \mathbf{y}, t ; z)$ is left invariant under replacing $t$ with $1 / t$ and $z$ with $t z$, while $\Xi^{-}(\mathbf{x}, \mathbf{y}, t ; z)$ is multiplied by $t$ after these substitutions, the coefficient of $z^{n}$ in $\Xi^{+}(\mathbf{x}, \mathbf{y}, t ; z)$ (respectively, $\left.\Xi^{-}(\mathbf{x}, \mathbf{y}, t ; z)\right)$ is a symmetric polynomial in $t$ with center of symmetry $n / 2$ (respectively, $(n-1) / 2$ ) for every $n \in \mathbb{N}$. Since the corresponding properties are clear for the coefficient of $z^{n}$ in the two summands in the right-hand side of Equation (28) and because of the uniqueness of the decomposition of a polynomial $f(t)$ as a sum of two symmetric polynomials with centers of symmetry $n / 2$ and $(n-1) / 2$ (see [5, Section 5.1]), we conclude that (8) and (9) follow from the single equation (28).

The second equalities in (26) and (27) follow by expanding $s_{\lambda}(\mathbf{x}) s_{\mu}(\mathbf{y})$ according to Equation (17) and then using the Robinson-Schensted correspondence of type $B$ and its properties $\operatorname{sDes}(w)=\operatorname{sDes}\left(Q^{B}(w)\right)$ and $\operatorname{Des}_{B}\left(w^{-1}\right)=\operatorname{Des}_{B}\left(\mathcal{P}^{B}(w)\right)$, exactly as in the proof of Corollary 4.1.

EXAmPle 4.5. The coefficient of $z^{2}$ in the left-hand side of Equations (8) and (9) equals

$$
\text { - } e_{1}(\mathbf{x}) e_{1}(\mathbf{y}) t+e_{2}(\mathbf{y}) t=s_{(1)}(\mathbf{x}) s_{(1)}(\mathbf{y}) t+s_{(1,1)}(\mathbf{y}) t \text {, and }
$$


- $e_{2}(\mathbf{x})(1+t)=s_{(1,1)}(\mathbf{x})(1+t)$,

respectively. Hence, $\xi_{2,0}^{+}(\mathbf{x}, \mathbf{y})=0, \xi_{2,1}^{+}(\mathbf{x}, \mathbf{y})=s_{(1)}(\mathbf{x}) s_{(1)}(\mathbf{y})+s_{(1,1)}(\mathbf{y})$ and $\xi_{2,0}^{-}(\mathbf{x}, \mathbf{y})=s_{(1,1)}(\mathbf{x})$, in agreement with Corollary 4.4 .

Proposition 4.6. For the $\mathcal{B}_{n}$-poset $s B_{n}$ we have

(29) $1+\sum_{n \geqslant 1} \operatorname{ch}_{\mathcal{B}}\left(\widetilde{H}_{n-1}\left(\left(s B_{n} * T_{t, n}\right)_{-} ; \mathbb{C}\right)\right) z^{n}=\frac{(1-t) E(\mathbf{y} ; z) E(\mathbf{x} ; t z) E(\mathbf{y} ; t z)}{E(\mathbf{x} ; t z) E(\mathbf{y} ; t z)-t E(\mathbf{x} ; z) E(\mathbf{y} ; z)}$

Proof. Following the reasoning in the proof of [17, Equation (3.3)], we set

$$
L_{n}(\mathbf{x}, \mathbf{y} ; t):=\operatorname{ch}_{\mathcal{B}}\left(L\left(\left(s B_{n} * T_{t, n}\right)_{-} ; \mathcal{B}_{n}\right)\right),
$$

where $L(P ; G)$ denotes the Lefschetz character of the $G$-poset $P$ over $\mathbb{C}$ (see Section 2 ). Since $\left(s B_{n} * T_{t, n}\right)_{-}$is Cohen-Macaulay over $\mathbb{C}$ of rank $n-1$, we have

$$
\operatorname{ch}_{\mathcal{B}}\left(\widetilde{H}_{n-1}\left(\left(s B_{n} * T_{t, n}\right)_{-} ; \mathbb{C}\right)\right)=(-1)^{n-1} L_{n}(\mathbf{x}, \mathbf{y} ; t) .
$$

Thus, the left-hand side of (29) is equal to $-\sum_{n \geqslant 0} L_{n}(\mathbf{x}, \mathbf{y} ; t)(-z)^{n}$. The sequence of posets $\left(s B_{0}, s B_{1}, \ldots, s B_{n}\right)$ can easily be verified to be $\left(\mathcal{B}_{0} \times \mathfrak{S}_{n}, \mathcal{B}_{1} \times \mathfrak{S}_{n-1}, \ldots, \mathcal{B}_{n} \times\right.$ $\mathfrak{S}_{0}$ )-uniform (see Section 2). Moreover, there is a single $\mathcal{B}_{n}$-orbit of elements of $s B_{n}$ of rank $k$ for each $k \in\{0,1, \ldots, n\}$. Thus, applying (13) to this sequence gives

$$
1_{\mathcal{B}_{n}} \oplus \bigoplus_{k=0}^{n}[k+1]_{t} L\left(\left(s B_{n-k} * T_{t, n-k}\right)_{-} ; \mathcal{B}_{n-k} \times \mathfrak{S}_{k}\right) \uparrow_{\mathcal{B}_{n-k} \times \mathfrak{S}_{k}}^{\mathcal{B}_{n}} \cong_{\mathcal{B}_{n}} 0 .
$$

Applying the characteristic map $\mathrm{ch}_{\mathcal{B}}$, as in the proof of Proposition 4.3, gives

$$
\sum_{k=0}^{n}[k+1]_{t} h_{k}(\mathbf{x}, \mathbf{y}) L_{n-k}(\mathbf{x}, \mathbf{y} ; t)=-h_{n}(\mathbf{x})
$$

Standard manipulation with generating functions, as in the proof of [17, Equation (3.3)], results in the formula

$$
\sum_{n \geqslant 0} L_{n}(\mathbf{x}, \mathbf{y} ; t) z^{n}=-\frac{H(\mathbf{x} ; z)}{\sum_{n \geqslant 0}[n+1]_{t} h_{n}(\mathbf{x}, \mathbf{y}) z^{n}}=-\frac{(1-t) H(\mathbf{x} ; z)}{H(\mathbf{x}, \mathbf{y} ; z)-t H(\mathbf{x}, \mathbf{y} ; t z)} .
$$

The proof now follows by switching $z$ to $-z$ and using the identities $E(\mathbf{x} ; z) H(\mathbf{x} ;-z)=$ 1 and $E(\mathbf{x}, \mathbf{y} ; z)=E(\mathbf{x} ; z) E(\mathbf{y} ; z)$.

Corollary 4.7. Equations (10) and (11) are valid for the functions

$$
\gamma_{n, k}^{+}(\mathbf{x}, \mathbf{y})=\sum_{(\lambda, \mu) \vdash n} d_{(\lambda, \mu), k}^{+} \cdot s_{\lambda}(\mathbf{x}) s_{\mu}(\mathbf{y})=\sum_{w} F_{\mathrm{sDes}(w)}(\mathbf{x}, \mathbf{y})
$$

and

$$
\gamma_{n, k}^{-}(\mathbf{x}, \mathbf{y})=\sum_{(\lambda, \mu) \vdash n} d_{(\lambda, \mu), k}^{-} \cdot s_{\lambda}(\mathbf{x}) s_{\mu}(\mathbf{y})=\sum_{w} F_{\mathrm{sDes}(w)}(\mathbf{x}, \mathbf{y}),
$$

where $d_{(\lambda, \mu), k}^{+}$(respectively, $d_{(\lambda, \mu), k}^{-}$) is the number of bitableaux $\mathcal{P} \in \operatorname{SYT}(\lambda, \mu)$ for which $\operatorname{Asc}_{B}(\mathcal{P}) \in \operatorname{Stab}([n])$ has $k$ elements and does not contain (respectively, contains) $n$ and, similarly, $w \in \mathcal{B}_{n}$ runs through all signed permutations for which $\operatorname{Asc}_{B}\left(w^{-1}\right) \in \operatorname{Stab}([n])$ has $k$ elements and does not contain (respectively, contains) $n$.

Proof. This statement follows by the same reasoning as in the proof of Corollary 4.4, provided one appeals to the first part of Theorem 1.1 and Proposition 4.6 instead. 
EXAMPLE 4.8. The coefficient of $z^{2}$ in the left-hand side of Equations (10) and (11) equals

- $e_{2}(\mathbf{x})\left(1+t+t^{2}\right)+e_{1}(\mathbf{x})^{2} t+e_{1}(\mathbf{x}) e_{1}(\mathbf{y}) t=s_{(1,1)}(\mathbf{x})(1+t)^{2}+s_{(2)}(\mathbf{x}) t+$ $s_{(1)}(\mathbf{x}) s_{(1)}(\mathbf{y}) t$

- $e_{1}(\mathbf{x}) e_{1}(\mathbf{y})\left(t+t^{2}\right)+e_{2}(\mathbf{y})\left(t+t^{2}\right)=s_{(1)}(\mathbf{x}) s_{(1)}(\mathbf{y}) t(1+t)+s_{(1,1)}(\mathbf{y}) t(1+t)$,

respectively. Hence, we have $\gamma_{2,0}^{+}(\mathbf{x}, \mathbf{y})=s_{(1,1)}(\mathbf{x}), \gamma_{2,1}^{+}(\mathbf{x}, \mathbf{y})=s_{(2)}(\mathbf{x})+s_{(1)}(\mathbf{x}) s_{(1)}(\mathbf{y})$ and $\gamma_{2,1}^{-}(\mathbf{x}, \mathbf{y})=s_{(1)}(\mathbf{x}) s_{(1)}(\mathbf{y})+s_{(2)}(\mathbf{y})$, in agreement with Corollary 4.7.

\section{An instance of the local equivariant Gal phenomenon}

This section uses Equation (8) to verify an equivariant analogue of Gal's conjecture [10] for the local face module of a certain triangulation of the simplex with interesting combinatorial properties. Background and any undefined terminology on simplicial complexes can be found in [24].

To explain the setup, let $V_{n}=\left\{\varepsilon_{1}, \varepsilon_{2}, \ldots, \varepsilon_{n}\right\}$ be the set of unit coordinate vectors in $\mathbb{R}^{n}$ and $\Sigma_{n}$ be the geometric simplex on the vertex set $V_{n}$. Consider a triangulation $\Gamma$ of $\Sigma_{n}$ (meaning, a geometric simplicial complex which subdivides $\Sigma_{n}$ ) with vertex set $V_{\Gamma}$ and the polynomial ring $S=\mathbb{C}\left[x_{v}: v \in V_{\Gamma}\right]$ in indeterminates which are in one-to-one correspondence with the vertices of $\Gamma$. The face ring [24, Chapter II] of $\Gamma$ is defined as the quotient ring $\mathbb{C}[\Gamma]=S / I_{\Gamma}$, where $I_{\Gamma}$ is the ideal of $S$ generated by the square-free monomials which correspond to the non-faces of $\Gamma$. Following $[23$, p. 824], we consider the linear forms

$$
\theta_{i}=\sum_{v \in V_{\Gamma}}\left\langle v, \varepsilon_{i}\right\rangle x_{v}
$$

for $i \in[n]$, where $\langle$,$\rangle is the standard inner product on \mathbb{R}^{n}$, and denote by $\Theta$ the ideal in $\mathbb{C}[\Gamma]$ generated by $\theta_{1}, \theta_{2}, \ldots, \theta_{n}$. This sequence is a special linear system of parameters for $\mathbb{C}[\Gamma]$, in the sense of [23, Definition 4.2]. As a result, the quotient ring $\mathbb{C}(\Gamma)=\mathbb{C}[\Gamma] / \Theta$ is a finite dimensional, graded $\mathbb{C}$-vector space and so is the local face module $L_{V_{n}}(\Gamma)$, defined [23, Definition 4.5] as the image in $\mathbb{C}(\Gamma)$ of the ideal of $\mathbb{C}[\Gamma]$ generated by the square-free monomials which correspond to the faces of $\Gamma$ lying in the interior of $\Sigma_{n}$. The Hilbert polynomials $\sum_{i=0}^{n} \operatorname{dim}_{\mathbb{C}}(\mathbb{C}(\Gamma))_{i} t^{i}$ and $\sum_{i=0}^{n} \operatorname{dim}_{\mathbb{C}}\left(L_{V_{n}}(\Gamma)\right)_{i} t^{i}$ of $\mathbb{C}(\Gamma)$ and $L_{V_{n}}(\Gamma)$ are two important enumerative invariants of $\Gamma$, namely the $h$-polynomial [24, Section II.2] and the local $h$-polynomial [23, Section 2] [24, Section III.10], respectively.

Suppose that $G$ is a subgroup of the automorphism group $\mathfrak{S}_{n}$ of $\Sigma_{n}$ which acts simplicially on $\Gamma$. Then, $G$ acts on the polynomial ring $S$ and (as discussed on [28, p. 250]) leaves the $\mathbb{C}$-linear span of $\theta_{1}, \theta_{2}, \ldots, \theta_{n}$ invariant. Therefore, $G$ acts on the graded $\mathbb{C}$-vector spaces $\mathbb{C}(\Gamma)$ and $L_{V_{n}}(\Gamma)$ as well and the polynomials $\sum_{i=0}^{n}(\mathbb{C}(\Gamma))_{i} t^{i}$ and $\sum_{i=0}^{n}\left(L_{V_{n}}(\Gamma)\right)_{i} t^{i}$, whose coefficients lie in the representation ring of $G$, are equivariant generalizations of the $h$-polynomial and local $h$-polynomial of $\Gamma$, respectively. The pair $(\Gamma, G)$ is said (see also [5, Section 5.2]) to satisfy the local equivariant Gal phenomenon if

$$
\sum_{i=0}^{n}\left(L_{V_{n}}(\Gamma)\right)_{i} t^{i}=\sum_{k=0}^{\lfloor n / 2\rfloor} M_{k} t^{k}(1+t)^{n-2 k}
$$

for some non-virtual $G$-representations $M_{k}$. This is an analogue for local face modules of the equivariant Gal phenomenon, formulated by Shareshian and Wachs [20, Section 5] for group actions on (flag) triangulations of spheres as an equivariant version of Gal's conjecture [10, Conjecture 2.1.7]. For trivial actions on flag triangulations of simplices, the validity of the local equivariant Gal phenomenon was conjectured in [2] 
and has been verified in many special cases; see [4, Section 4] [5, Section 3.2] and references therein.

Although it would be too optimistic to expect that the local equivariant Gal phenomenon holds for all group actions on flag triangulations of $\Sigma_{n}$, the case $G=\mathfrak{S}_{n}$ deserves special attention. We then use the notation

$$
\begin{aligned}
\operatorname{ch}(\mathbb{C}(\Gamma), t) & :=\sum_{i=0}^{n} \operatorname{ch}(\mathbb{C}(\Gamma))_{i} t^{i}, \\
\operatorname{ch}\left(L_{V_{n}}(\Gamma), t\right) & :=\sum_{i=0}^{n} \operatorname{ch}\left(L_{V_{n}}(\Gamma)\right)_{i} t^{i} .
\end{aligned}
$$

For the (first) barycentric subdivision of $\Sigma_{n}$ we have the following result of Stanley.

Proposition 5.1 ([23, Proposition 4.20]). For the $\mathfrak{S}_{n}$-action on the barycentric subdivision $\Gamma_{n}$ of the simplex $\Sigma_{n}$, we have

$$
1+\sum_{n \geqslant 1} \operatorname{ch}\left(L_{V_{n}}\left(\Gamma_{n}\right), t\right) z^{n}=\frac{1-t}{H(\mathbf{x} ; t z)-t H(\mathbf{x} ; z)} .
$$

Combining this result with Gessel's identity (6) gives

$$
\operatorname{ch}\left(L_{V_{n}}\left(\Gamma_{n}\right), t\right)=\sum_{k=0}^{\lfloor(n-2) / 2\rfloor} \omega \xi_{n, k}(\mathbf{x}) t^{k+1}(1+t)^{n-2 k-2},
$$

where $\omega$ is the standard involution on symmetric functions exchanging $e_{\lambda}(\mathbf{x})$ and $h_{\lambda}(\mathbf{x})$ for every $\lambda$, whence it follows that $\left(\Gamma_{n}, \mathfrak{S}_{n}\right)$ satisfies the local equivariant Gal phenomenon for every $n$.

The combinatorics of the barycentric subdivision $\Gamma_{n}$ is related to the symmetric group $\mathfrak{S}_{n}$. We now consider a triangulation $K_{n}$ of the simplex $\Sigma_{n}$, studied in [16, Chapter 3] (see also [4, Remark 4.5] [5, Section 3.3]) and shown on the right of Figure 1 for $n=3$, the combinatorics of which is related to the hyperoctahedral group $\mathcal{B}_{n}$. The triangulation $K_{n}$ can be defined as the barycentric subdivision of the standard cubical subdivision of $\Sigma_{n}$, shown on the left of Figure 1 for $n=3$, whose faces are in inclusion-preserving bijection with the nonempty closed intervals in the truncated Boolean lattice $B_{n} \backslash\{\varnothing\}$. Thus, the faces of $K_{n}$ correspond bijectively to chains of nonempty closed intervals in $B_{n} \backslash\{\varnothing\}$ and $\mathfrak{S}_{n}$ acts simplicially on $K_{n}$ in the obvious way. As a simplicial complex, $K_{n}$ can be thought of as a "half Coxeter complex" for $\mathcal{B}_{n}$.
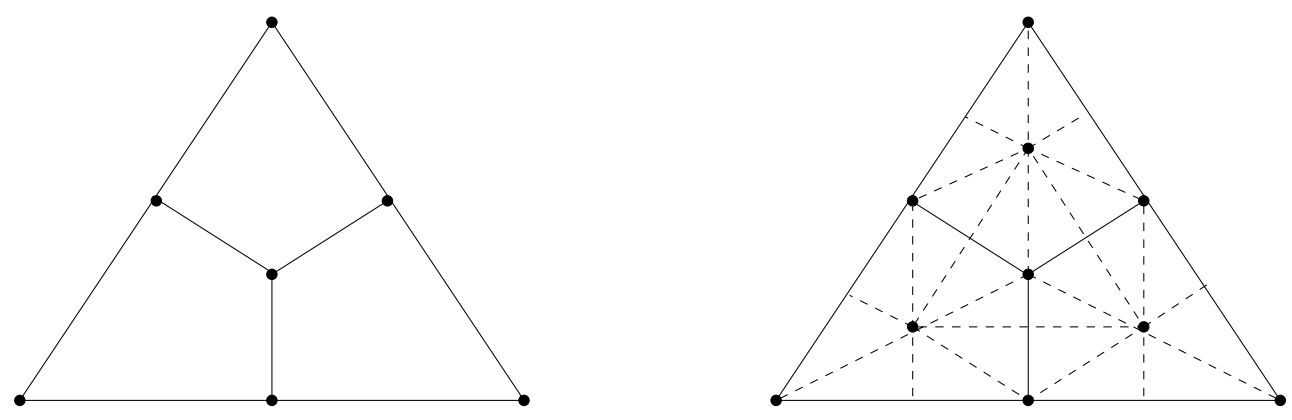

Figure 1. The triangulation $K_{3}$ 
Proposition 5.2. For the $\mathfrak{S}_{n}$-action on $K_{n}$ we have

$$
1+\sum_{n \geqslant 1} \operatorname{ch}\left(\mathbb{C}\left(K_{n}\right), t\right) z^{n}=\frac{H(\mathbf{x} ; z)(H(\mathbf{x} ; t z)-t H(\mathbf{x} ; z))}{H(\mathbf{x} ; t z)^{2}-t H(\mathbf{x} ; z)^{2}}
$$

and

$$
1+\sum_{n \geqslant 1} \operatorname{ch}\left(L_{V_{n}}\left(K_{n}\right), t\right) z^{n}=\frac{H(\mathbf{x} ; t z)-t H(\mathbf{x} ; z)}{H(\mathbf{x} ; t z)^{2}-t H(\mathbf{x} ; z)^{2}} .
$$

Moreover, the pair $\left(K_{n}, \mathfrak{S}_{n}\right)$ satisfies the local equivariant Gal phenomenon for every $n$.

The proof relies on methods developed by Stembridge [28] to study representations of Weyl groups on the cohomology of the toric varieties associated to Coxeter complexes. To prepare for it, we recall that the $h$-polynomial of a simplicial complex $\Delta$ of dimension $n-1$ is defined as

$$
h(\Delta, t)=\sum_{i=0}^{n} f_{i-1}(\Delta) t^{i}(1-t)^{n-i}
$$

where $f_{i}(\Delta)$ stands for the number of $i$-dimensional faces of $\Delta$. Consider a pair $(\Gamma, G)$, consisting of a triangulation $\Gamma$ of $\Sigma_{n}$ and a subgroup $G$ of $\mathfrak{S}_{n}$ acting on $\Gamma$, as discussed earlier. Following [28, Section 1], we call the action of $G$ on $\Gamma$ proper if $w$ fixes all vertices of every face $F \in \Delta$ which is fixed by $w$, for every $w \in G$. Note that group actions, such as the $\mathfrak{S}_{n}$-actions on $\Gamma_{n}$ and $K_{n}$, on the order complex (simplicial complex of chains) of a poset which are induced by an action on the poset itself, are always proper. Under this assumption, the set $\Gamma^{w}$ of faces of $\Gamma$ which are fixed by $w$ forms an induced subcomplex of $\Gamma$, for every $w \in G$.

Although Stembridge [28] deals with triangulations of spheres, rather than simplices, his methods apply to our setting and his Theorem 1.4, combined with the considerations of Section 6 in [28], imply that

$$
\operatorname{ch}(\mathbb{C}(\Gamma), t)=\frac{1}{n !} \sum_{w \in \mathfrak{S}_{n}} \frac{h\left(\Gamma^{w}, t\right)}{(1-t)^{1+\operatorname{dim}\left(\Gamma^{w}\right)}} \prod_{i \geqslant 1}\left(1-t^{\lambda_{i}(w)}\right) p_{\lambda_{i}(w)}(\mathbf{x})
$$

for every proper $\mathfrak{S}_{n}$-action on $\Gamma$, where $\lambda_{1}(w) \geqslant \lambda_{2}(w) \geqslant \cdots$ are the sizes of the cycles of $w \in \mathfrak{S}_{n}$ and $p_{k}(\mathbf{x})$ is a power sum symmetric function.

Proof of Proposition 5.2. To prove Equation (35), we follow the analogous computation in [28, Section 6] for the barycentric subdivision of the boundary complex of the simplex. We first note that $\left(K_{n}\right)^{w}$ is combinatorially isomorphic to $K_{c(w)}$ for every $w \in \mathfrak{S}_{n}$, where $c(w)$ is the number of cycles of $w$. Furthermore, it was shown in [16, Section 3.6] that, in the notation of Section $3, h\left(K_{n}, t\right)$ is the "half $\mathcal{B}_{n}$-Eulerian polynomial"

$$
B_{n}^{+}(t)=\sum_{w \in \mathcal{B}_{n}^{+}} t^{\left|\operatorname{Des}_{B}(w)\right|},
$$

where $\mathcal{B}_{n}^{+}$consists of the signed permutations $w \in \mathcal{B}_{n}$ with negative first coordinate. These remarks and Equation (37) imply that

$$
\operatorname{ch}\left(\mathbb{C}\left(K_{n}\right), t\right) z^{n}=\sum_{\lambda=\left(\lambda_{1}, \lambda_{2}, \ldots\right) \vdash n} m_{\lambda}^{-1} \frac{B_{\ell(\lambda)}^{+}(t)}{(1-t)^{\ell(\lambda)}} \prod_{i \geqslant 1}\left(1-t^{\lambda_{i}}\right) p_{\lambda_{i}}(\mathbf{x}) z^{\lambda_{i}},
$$

where $n ! / m_{\lambda}$ is the cardinality of the conjugacy class of $\mathfrak{S}_{n}$ which corresponds to $\lambda \vdash n$ and $\ell(\lambda)$ is the number of parts of $\lambda$. The polynomials $B_{n}^{+}(t)$ are known (see, 
for instance, [16, Equation 3.7.5]) to satisfy

$$
\frac{B_{n}^{+}(t)}{(1-t)^{n}}=\sum_{k \geqslant 0}\left((2 k+1)^{n}-(2 k)^{n}\right) t^{k}
$$

and hence, we may rewrite the previous formula as

$$
\begin{aligned}
\operatorname{ch}( & \left.\mathbb{C}\left(K_{n}\right), t\right) z^{n} \\
& =\sum_{k \geqslant 0} t^{k} \sum_{\lambda=\left(\lambda_{1}, \lambda_{2}, \ldots\right) \vdash n} m_{\lambda}^{-1}\left((2 k+1)^{\ell(\lambda)}-(2 k)^{\ell(\lambda)}\right) \prod_{i \geqslant 1}\left(1-t^{\lambda_{i}}\right) p_{\lambda_{i}}(\mathbf{x}) z^{\lambda_{i}} .
\end{aligned}
$$

Summing over all $n \geqslant 1$ and using the standard identities

$$
H(\mathbf{x} ; z)=\sum_{\lambda} m_{\lambda}^{-1} p_{\lambda}(\mathbf{x}) z^{|\lambda|}=\exp \left(\sum_{n \geqslant 1} p_{n}(\mathbf{x}) z^{n} / n\right)
$$

just as in the proof of [28, Theorem 6.2] (one considers the $p_{n}$ as algebraically independent indeterminates and replaces first each $p_{n}$ with $(2 k+1)\left(1-t^{n}\right) p_{n}$, then with $\left.(2 k)\left(1-t^{n}\right) p_{n}\right)$, we conclude that

$$
\begin{aligned}
1+\sum_{n \geqslant 1} \operatorname{ch}\left(\mathbb{C}\left(K_{n}\right), t\right) z^{n} & =1+\sum_{k \geqslant 0} t^{k}\left(\frac{H(\mathbf{x} ; z)^{2 k+1}}{H(\mathbf{x} ; t z)^{2 k+1}}-\frac{H(\mathbf{x} ; z)^{2 k}}{H(\mathbf{x} ; t z)^{2 k}}\right) \\
& =1+\left(\frac{H(\mathbf{x} ; z)}{H(\mathbf{x} ; t z)}-1\right)\left(1-t \frac{H(\mathbf{x} ; z)^{2}}{H(\mathbf{x} ; t z)^{2}}\right)^{-1} \\
& =\frac{H(\mathbf{x} ; z)(H(\mathbf{x} ; t z)-t H(\mathbf{x} ; z))}{H(\mathbf{x} ; t z)^{2}-t H(\mathbf{x} ; z)^{2}}
\end{aligned}
$$

and the proof of (35) follows. To prove (36), it suffices to observe that

$$
1+\sum_{n \geqslant 1} \operatorname{ch}\left(L_{V_{n}}\left(K_{n}\right), t\right) z^{n}=E(\mathbf{x},-z)\left(1+\sum_{n \geqslant 1} \operatorname{ch}\left(\mathbb{C}\left(K_{n}\right), t\right) z^{n}\right) .
$$

The latter follows exactly as the corresponding identity for the barycentric subdivision $\Gamma_{n}$, shown in the proof of [23, Proposition 4.20]. Finally, from Equations (8) and (36) we deduce that

$$
\operatorname{ch}\left(L_{V_{n}}\left(K_{n}\right), t\right)=\sum_{k=0}^{\lfloor n / 2\rfloor} \omega \xi_{n, k}^{+}(\mathbf{x}, \mathbf{x}) t^{k}(1+t)^{n-2 k}
$$

This expression, Corollary 4.4 and the well known fact that $s_{\lambda}(\mathbf{x}) s_{\mu}(\mathbf{x})$ is Schurpositive for all partitions $\lambda, \mu$ imply that $\operatorname{ch}\left(L_{V_{n}}\left(K_{n}\right), t\right)$ is Schur $\gamma$-positive for every $n$, as claimed in the last statement of the proposition.

\section{An instance of the EQUivariant GAL PHENOMENON}

A very interesting group action on a simplicial complex is that of a finite Coxeter group $W$ on its Coxeter complex [7]. When $W$ is crystallographic, this action induces a graded $W$-representation on the (even degree) cohomology of the associated projective toric variety which has been studied by Procesi [14], Stanley [22, p. 529], Dolgachev and Lunts [9], Stembridge [28] and Lehrer [11], among others. The graded dimension of this representation is equal to the $W$-Eulerian polynomial. Its equivariant $\gamma$-positivity is a consequence of a variant of Equation (7) in the case of the symmetric group $\mathfrak{S}_{n}$; see [5, Section 5] [20, Section 5]. In the case of the hyperoctahedral group $\mathcal{B}_{n}$, by 
[9, Theorem 6.3] or [28, Theorem 7.6], the Frobenius characteristic of this graded $\mathcal{B}_{n}$-representation is equal to the coefficient of $z^{n}$ in

$$
\frac{(1-t) H(\mathbf{x} ; z) H(\mathbf{x} ; t z)}{H(\mathbf{x}, \mathbf{y} ; t z)-t H(\mathbf{x}, \mathbf{y} ; z)} .
$$

The following statement (and its proof) shows that the equivariant $\gamma$-positivity of this graded representation is a consequence of the results of Section 4 and confirms another instance of the equivariant Gal phenomenon of Shareshian and Wachs. As discussed in [5, Section 5], it is reasonable to expect that the same holds for the action of any finite crystallographic Coxeter group $W$ on its Coxeter complex; that would provide a natural equivariant analogue to the $\gamma$-positivity of $W$-Eulerian polynomials [ 5 , Section 2.1.3].

Proposition 6.1. The coefficient of $z^{n}$ in (40) is Schur $\gamma$-positive for every $n \in \mathbb{N}$.

Proof. Using Equations (6) with (10) we find that

$$
\begin{aligned}
\frac{(1-t) E(\mathbf{x} ; z) E(\mathbf{x} ; t z)}{E(\mathbf{x}, \mathbf{y} ; t z)-t E(\mathbf{x}, \mathbf{y} ; t z)}= & \frac{E(\mathbf{x} ; z) E(\mathbf{x} ; t z)(E(\mathbf{y} ; t z)-t E(\mathbf{y} ; z))}{E(\mathbf{x} ; t z) E(\mathbf{y} ; t z)-t E(\mathbf{x} ; z) E(\mathbf{y} ; t z)} \cdot \frac{1-t}{E(\mathbf{y} ; t z)-t E(\mathbf{y} ; z)} \\
= & \left(\sum_{n \geqslant 0} z^{n} \sum_{i=0}^{\lfloor n / 2\rfloor} \gamma_{n, i}^{+}(\mathbf{x}, \mathbf{y}) t^{i}(1+t)^{n-2 i}\right) \\
& \cdot\left(1+\sum_{n \geqslant 2} z^{n} \sum_{j=1}^{\lfloor n / 2\rfloor} \xi_{n, j-1}(\mathbf{y}) t^{j}(1+t)^{n-2 j}\right) \\
= & \sum_{n \geqslant 0} z^{n} \sum_{k+\ell=n} \sum_{i, j} \gamma_{k, i}^{+}(\mathbf{x}, \mathbf{y}) \xi_{\ell, j-1}(\mathbf{y}) t^{i+j}(1+t)^{n-2 i-2 j},
\end{aligned}
$$

where we have set $\xi_{0,-1}(\mathbf{y}):=1$ and $\xi_{1,-1}(\mathbf{y}):=0$. Since Schur-positivity is preserved by sums, products and the standard involution on symmetric functions, this computation implies that the coefficient of $z^{n}$ in (40) is Schur $\gamma$-positive for every $n \in \mathbb{N}$ and the proof follows.

REMARK 6.2. We have shown that

$$
\frac{(1-t) H(\mathbf{x} ; z) H(\mathbf{x} ; t z)}{H(\mathbf{x}, \mathbf{y} ; t z)-t H(\mathbf{x}, \mathbf{y} ; z)}=1+\sum_{n \geqslant 1} z^{n} \sum_{i=0}^{\lfloor n / 2\rfloor} \gamma_{n, i}^{B}(\mathbf{x}, \mathbf{y}) t^{i}(1+t)^{n-2 i}
$$

for some Schur-positive symmetric functions $\gamma_{n, i}^{B}(\mathbf{x}, \mathbf{y})$. It is an open problem to find an explicit combinatorial interpretation of the coefficient $c_{(\lambda, \mu), i}^{B}$ of $s_{\lambda}(\mathbf{x}) s_{\mu}(\mathbf{y})$ in $\gamma_{n, i}^{B}(\mathbf{x}, \mathbf{y})$, for $(\lambda, \mu) \vdash n$. Comparing the graded dimensions of the $\mathcal{B}_{n}$-representations whose Frobenius characteristic is given by the two sides of Equation (41) we get

$$
B_{n}(t)=\sum_{(\lambda, \mu) \vdash n}\left(\begin{array}{c}
n \\
|\lambda|
\end{array}\right) f^{\lambda} f^{\mu} \sum_{i=0}^{\lfloor n / 2\rfloor} c_{(\lambda, \mu), i}^{B} t^{i}(1+t)^{n-2 i},
$$

where $B_{n}(t):=\sum_{w \in \mathcal{B}_{n}} t^{\left|\operatorname{Des}_{B}(w)\right|}$ is the $\mathcal{B}_{n}$-Eulerian polynomial and $f^{\lambda}$ stands for the number of standard Young tableaux of shape $\lambda$. Thus, a solution to this problem would provide a refinement of the known $\gamma$-positivity of $\mathcal{B}_{n}$-Eulerian polynomials; see [5, Section 2.1.3].

Acknowledgements. The author wishes to thank Eric Katz and Kalle Karu for some helpful e-discussions about the local equivariant Gal phenomenon and the anonymous referees for their useful comments. 


\section{REFERENCES}

[1] Ron M. Adin, Christos A. Athanasiadis, Sergi Elizalde, and Yuval Roichman, Character formulas and descents for the hyperoctahedral group, Adv. in Appl. Math. 87 (2017), 128-169.

[2] Christos A. Athanasiadis, Flag subdivisions and $\gamma$-vectors, Pacific J. Math. 259 (2012), no. 2, $257-278$.

[3], Edgewise subdivisions, local h-polynomials, and excedances in the wreath product $\mathbb{Z}_{r}$ ? $\mathfrak{S}_{n}$, SIAM J. Discrete Math. 28 (2014), no. 3, 1479-1492.

[4] A survey of subdivisions and local h-vectors, in The mathematical legacy of Richard P. Stanley, Amer. Math. Soc., Providence, RI, 2016, pp. 39-51.

[5] _ Gamma-positivity in combinatorics and geometry, Sém. Lothar. Combin. 77 ([20162018]), B77i (64 pages).

[6] Binomial Eulerian polynomials for colored permutations, https://arxiv.org/abs/ $1812.00434,2018$

[7] Anders Björner, Some combinatorial and algebraic properties of Coxeter complexes and Tits buildings, Adv. Math. 52 (1984), no. 3, 173-212.

[8] Anders Björner and Volkmar Welker, Segre and Rees products of posets, with ring-theoretic applications, J. Pure Appl. Algebra 198 (2005), no. 1-3, 43-55.

[9] Igor Dolgachev and Valery Lunts, A character formula for the representation of a Weyl group in the cohomology of the associated toric variety, J. Algebra 168 (1994), no. 3, 741-772.

[10] Światosław R. Gal, Real root conjecture fails for five- and higher-dimensional spheres, Discrete Comput. Geom. 34 (2005), no. 2, 269-284.

[11] Gustav I. Lehrer, Rational points and Coxeter group actions on the cohomology of toric varieties, Ann. Inst. Fourier (Grenoble) 58 (2008), no. 2, 671-688.

[12] Svante Linusson, John Shareshian, and Michelle L. Wachs, Rees products and lexicographic shellability, J. Comb. 3 (2012), no. 3, 243-276.

[13] Stéphane Poirier, Cycle type and descent set in wreath products, Proceedings of the 7th Conference on Formal Power Series and Algebraic Combinatorics (Noisy-le-Grand, 1995), vol. 180, Discrete Math., no. 1-3, 1998, pp. 315-343.

[14] Claudio Procesi, The toric variety associated to Weyl chambers, in Mots, Lang. Raison. Calc. Hermès, Paris, 1990, pp. 153-161.

[15] Bruce E. Sagan, The symmetric group, second ed., Graduate Texts in Mathematics, vol. 203, Springer-Verlag, New York, 2001.

[16] Christina Savvidou, Barycentric subdivisions, clusters and permutation enumeration (in Greek), Ph.D. thesis, University of Athens (Greece), 2013.

[17] John Shareshian and Michelle L. Wachs, Poset homology of Rees products, and q-Eulerian polynomials, Electron. J. Combin. 16 (2009), no. 2, Research Paper R20 (29 pages), Special volume in honor of Anders Björner.

[18] _ Eulerian quasisymmetric functions, Adv. Math. 225 (2010), no. 6, 2921-2966.

[19] _ From poset topology to q-Eulerian polynomials to Stanley's chromatic symmetric functions, in The mathematical legacy of Richard P. Stanley, Amer. Math. Soc., Providence, RI, 2016, pp. 301-321.

[20] , Gamma-positivity of variations of Eulerian polynomials, https://arxiv.org/abs/ $1702.06666 \mathrm{v} 3,2018$.

[21] Richard P. Stanley, Some aspects of groups acting on finite posets, J. Combin. Theory Ser. A 32 (1982), no. 2, 132-161.

$[22] \ldots$, Log-concave and unimodal sequences in algebra, combinatorics, and geometry, in Graph theory and its applications: East and West (Jinan, 1986), Ann. New York Acad. Sci., vol. 576, New York Acad. Sci., New York, 1989, pp. 500-535.

[23] Subdivisions and local h-vectors, J. Amer. Math. Soc. 5 (1992), no. 4, 805-851.

[24] _ Combinatorics and commutative algebra, second ed., Progress in Mathematics, vol. 41, Birkhäuser Boston, Inc., Boston, MA, 1996.

[25] _ Enumerative combinatorics. Vol. 2, Cambridge Studies in Advanced Mathematics, vol. 62, Cambridge University Press, Cambridge, 1999.

[26] __ Enumerative combinatorics. Volume 1, second ed., Cambridge Studies in Advanced Mathematics, vol. 49, Cambridge University Press, Cambridge, 2012.

[27] John R. Stembridge, The projective representations of the hyperoctahedral group, J. Algebra 145 (1992), no. 2, 396-453.

[28] Some permutation representations of Weyl groups associated with the cohomology of toric varieties, Adv. Math. 106 (1994), no. 2, 244-301. 
Christos A. Athanasiadis

[29] Sheila Sundaram, The homology representations of the symmetric group on Cohen-Macaulay subposets of the partition lattice, Adv. Math. 104 (1994), no. 2, 225-296.

[30] Michelle L. Wachs, Poset topology: tools and applications, in Geometric combinatorics, IAS/Park City Math. Ser., vol. 13, Amer. Math. Soc., 2007, pp. 497-615.

Christos A. Athanasiadis, Department of Mathematics, National and Kapodistrian University of Athens, Panepistimioupolis, Athens 15784, Greece

E-mail : caath@math.uoa.gr 